\title{
The null ideal restricted to some non-null set may be $\aleph_{1}$-saturated
}

\author{
by
}

\section{Saharon Shelah (Jerusalem and Piscataway, NJ)}

\begin{abstract}
Our main result is that possibly some non-null set of reals cannot be divided into uncountably many non-null sets. We also deal with a non-null set of reals, the graph of any function from which is null, and deal with our iterations somewhat more generally.
\end{abstract}

\section{Annotated content}

0. Introduction. We review results and background, and give notation.

1. The null ideal restricted to a non-null set may be $\aleph_{1}$-saturated. We explain the difficulty for the null case, solved by adding $\kappa$ random reals $\eta_{\lambda+\alpha}$ in the end, $\kappa$ measurable, but they are random over some subuniverses, so we add at the beginning $\lambda$ Cohens $\underline{\sim}_{i}(i<\lambda)$. The memory is devised such that $\left\{A:\left\{\eta_{\lambda+\alpha}: \alpha \in A\right\}\right.$ null $\}$ will include

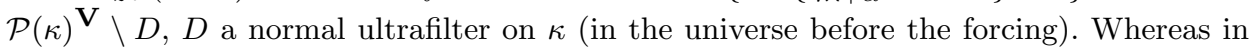
[Sh 592], the $A_{\alpha}$ (memory) were chosen closed enough, here we use automorphisms of the memory structure.

2. Non-null set with no non-null function. We show that consistently for some non-null set $A$ of reals for every function from $A$ to the reals, its graph is a null subset of the plane.

3. The $\mathbb{L}_{\aleph_{1}, \aleph_{1}}$-elementary submodels and the forcing. We deal with general FS iterations of c.c.c. definable in subuniverses, and give sufficient condition for $\mathbb{P}_{A}^{\prime} \lessdot \mathbb{P}_{\alpha}$. As application we show consistency of some values of $2^{\aleph_{0}}$, add(meagre), cov(meagre), unif(meagre), $\mathfrak{b}, \mathfrak{d}$.

2000 Mathematics Subject Classification: 03E35, 03E55, 03E19.

Key words and phrases: set theory, forcing, FS iteration, null ideal, measure, $\aleph_{1^{-}}$ saturated ideal, null functions, nep forcing.

Research supported by the Israel Science Foundation. Publication 619.

I would like to thank Alice Leonhardt for the beautiful typing. 
0. Introduction. Note that the result stated in the abstract tells us that the positive result explained below cannot be improved (to $\aleph_{1}$ sets). It is (Gitik and Shelah [GiSh:582])

(*) given sets $A_{n}$ of reals for $n<\omega$, we can find $B_{n} \subseteq A_{n}$ pairwise disjoint such that $A_{n}, B_{n}$ have the same outer Lebesgue measure.

Lately, we have proved ([Sh 592]):

0.1. Theorem. $\operatorname{Con}\left(\operatorname{cov}(\right.$ null $\left.)=\aleph_{\omega}+\mathrm{MA}_{\aleph_{n}}\right)$ for each $n<\omega$.

The idea of the proof was to use finite support $\left\langle\mathbb{P}_{i}, \mathbb{Q}_{i}: i<\alpha\right\rangle$, where say $\mathbb{Q}_{i}$ has generic real $r_{i}$ and $\mathbb{Q}_{i}$ is random forcing in $\left.\left.\mathbf{V} \widetilde{\langle} \underline{\sim}_{j}: j \in a_{i}\right\rangle\right], a_{i} \subseteq i$, $\widetilde{a}_{i}$ closed enough or ${\underset{\sim}{i}}_{i}$ is Cohen real but the "memory" is not transitive, i.e., in general $j \in a_{i} \nRightarrow a_{j} \subseteq a_{i}$.

As 0.1 was hard for me for long it seems reasonable to hope the solution will open my eyes on other problems as well.

In this paper we deal mainly with "can every non-null set be partitioned to uncountably many non-null sets?", equivalently: "can the ideal of null sets which are subsets of a fixed non-null subset of $\mathbb{R}$ be $\aleph_{1}$-saturated?". P. Komjáth $[\mathrm{Ko}]$ proved that it is consistent that there is a non-meagre set $A$ such that the ideal of meagre subsets of $A$ is $\aleph_{1}$-saturated. The question whether a similar fact may hold for measure dates back to Ulam (see also Prikry's thesis; Fremlin asked both versions in the seventies).

So we prove the following:

0.2. TheOREM. It is consistent that there is a non-null set $A \subseteq \mathbb{R}$ such that the ideal of null subsets of $A$ is $\aleph_{1}$-saturated (of course, provided that "ZFC $+\exists$ measurable" is consistent).

The proof of 0.1 was not directly applicable, but "turning the tables" make it relevant as explained in $\S 1$.

The question appears on Fremlin's current list of problems [Fe94] as problem EL(a).

We also have some further remarks, e.g. the exact cardinal assumption for 0.1 . We try to make the paper self-contained for readers with basic knowledge of forcing and of [Sh 592].

Also we answer the following problem which Komjáth draws our attention to:

\subsection{Theorem. It is consistent that}

$\oplus \quad$ there is a non-null $A \subseteq \mathbb{R}$ such that for every $f: A \rightarrow \mathbb{R}$, the function $f$ is null as a subset of the plane $\mathbb{R} \times \mathbb{R}$

provided that "ZFC+ there is a measurable cardinal" is consistent. 
Lastly, in $\S 3$ we investigate when partial memory iteration behaves as in [Sh 592], and give an example how to apply the method. This paper originally was a part of [Sh 592], but as the main part of [Sh 592] was in final form and this was not and we wanted to add $\S 3$, we separated.

0.4. Notation. (A) We denote:

(1) natural numbers by $k, l, m, n$ and also $i, j$,

(2) ordinals by $\alpha, \beta, \gamma, \delta, \zeta, \xi$ ( $\delta$ always limit),

(3) cardinals by $\lambda, \kappa, \chi, \mu$,

(4) reals by $a, b$ and positive reals (normally small) by $\varepsilon$,

(5) subsets of $\omega$ or ${ }^{\omega} \geq 2$ or Ord by $A, B, C, X, Y, Z$,

(6) Borel functions by $\mathcal{B}$,

(7) finitely additive measures by $\Xi$,

(8) sequences of natural numbers or ordinals by $\eta, \nu, \varrho$,

(9) various things by $s$.

(B) $\mathcal{T}$ is as in Definition 2.9 of [Sh 592], $t$ is a member of $\mathcal{T}$.

(C) We denote

(1) forcing notions by $\mathbb{P}, \mathbb{Q}$,

(2) forcing conditions by $p, q$,

(3) " $p$ stronger than $q$ " by $p \geq q$

and use $r$ to denote members of Random (see below).

(E)(1) Leb is Lebesgue measure (on $\left\{A: A \subseteq{ }^{\omega} 2\right\}$ ).

(2) Random will be the family

$\left\{r \subseteq{ }^{\omega>} 2: r\right.$ is a subtree of $\left({ }^{\omega>} 2, \triangleleft\right)$, i.e., closed under initial segments,

\langle\rangle$\in r$, with no $\triangleleft$-maximal element $\left(\operatorname{so} \lim (r) \subseteq{ }^{\omega} 2\right.$

is closed), $\operatorname{Leb}(\lim (r))>0$, and moreover,

$\eta \in r \Rightarrow \lim \left(r^{[\eta]}\right)$ is not null (on $r^{[\eta]}$ see below) $\}$

ordered by inverse inclusion. We may sometimes use instead

$\left\{B: B\right.$ is a Borel non-null subset of $\left.{ }^{\omega} 2\right\}$.

(F) For $\eta \in{ }^{\omega>} 2$ and $A \subseteq{ }^{\omega \geq} 2$ let

$$
A^{[\eta]}=\{\nu \in A: \nu \triangleleft \eta \vee \eta \unlhd \nu\} .
$$

We thank Tomek Bartoszyński, Mariusz Rabus and Heike Mildenberger for reading, commenting and suggesting corrections.

1. The null ideal restricted to a non-null set may be $\aleph_{1}$-saturated. Let us first describe an outline of Komjáth's solution to the problem for the meagre ideal. Note that by a theorem of Solovay, the conclusion 
implies that there is a measurable cardinal in some inner model. So Komjáth starts with

$$
\mathbf{V} \models \text { " } \kappa \text { is measurable and } D \text { is a normal ultrafilter on } \kappa \text { ". }
$$

He uses a finite support iteration $\left\langle\mathbb{P}_{\alpha}, \mathbb{Q}_{\beta}: \alpha \leq 2^{\kappa}, \beta<2^{\kappa}\right\rangle$ of c.c.c. forcing notions such that for $\beta<\kappa$ the forcing notion $\mathbb{Q}_{\beta}$ adds a Cohen real $\eta_{\beta} \in{ }^{\omega} 2$ (so $\mathbb{Q}_{\beta}=(\omega>2, \triangleleft)$ ) and for each $\beta \in\left[\kappa, 2^{\kappa}\right)$ for some $A_{\beta} \in D$ (so from $\mathbf{V}$ ), the forcing notion $\mathbb{Q}_{\beta}$ makes the set $\left\{\eta_{\gamma}: \gamma \in \kappa \backslash A_{\beta}\right\}$ meagre (and every $A \in D$ appears). The point is that finite support iterations tend to preserve non-meagreness, so in $\mathbf{V}^{\mathbb{P}_{2}}$ the set $\left\{\eta_{\alpha}: \alpha<\kappa\right\}$ remains non-meagre and as $\mathbb{P}_{2^{\kappa}}$ is a c.c.c. forcing notion, $D$ is (i.e. generates) in $\mathbf{V}^{\mathbb{P}_{2^{\kappa}}}$ an $\aleph_{1}$-saturated filter.

For our aims this per se is doomed to failure: finite support iterations add Cohen reals which make the set of old reals null, whereas countable support iterations tend to collapse $\aleph_{2}$ and we no longer know that $D$ generates an $\aleph_{1}$-saturated filter. This seems to indicate that the solution should be delayed till we have better other support iterations (the $2^{\aleph_{0}}=\aleph_{3}$ problem; see [Sh 666], [Sh:b, Chs. VII, VIII] and [Sh:f, Chs. VII, VIII]). But we start with a simpler remedy: we use a finite support iteration which illustrates a quotation from Lewis Caroll: the punishment will precede the sin, i.e., we first add the Cohens to make the required sets of reals null and only then do we add the $\kappa$ randoms which form the non-null set. That is, our iteration of c.c.c. forcing notions is $\left\langle\mathbb{P}_{\alpha}, \mathbb{Q}_{\beta}: \alpha \leq \delta^{*}, \beta<\delta^{*}\right\rangle$ with $\operatorname{cf}\left(\delta^{*}\right)=\kappa,\left\langle\beta_{\xi}: \xi<\kappa\right\rangle$ is increasing with limit $\delta^{*}, \mathbb{Q}_{\beta_{\xi}}$ is a partial random, say Random $\mathbf{v}^{\mathbb{P}_{A\left(\beta_{\xi}\right)}}$ where $A\left(\beta_{\xi}\right)=A_{\beta_{\xi}} \subseteq \beta_{\xi}$, adding the real $\mathcal{\tau}_{\xi}$, so actually $\mathbf{V}^{\left.\mathbb{P}_{A\left(\beta_{\xi}\right)}\right)}=\mathbf{V}\left[\left\langle\tau_{\gamma}: \gamma \in A_{\beta_{\xi}}\right\rangle\right]$. E.g. $\delta^{*}=\lambda+\kappa, \beta_{\xi}=\lambda+\xi$.

Clearly we would like to have

$$
\models_{\mathbb{P}_{\delta^{*}}} \text { " }\left\{\tau_{\beta_{\xi}}: \xi<\kappa\right\} \text { is not null". }
$$

For this it suffices to have: every countable $A \subseteq \delta^{*}$ is included in some $A\left(\beta_{\xi}\right)$. However, we would also like to have the set $\left\{\tau_{\beta_{\xi}}: \xi \in \kappa \backslash B\right\}$ null for every $B \in D$. For this it is natural to demand that for some $\alpha, \underset{\sim}{\mathbb{Q}}$ is Cohen forcing and

$$
(\forall \xi \in \kappa \backslash B)\left(\alpha \notin A_{\beta_{\xi}}\right) .
$$

So we try to force a null set including $\left\{\mathcal{\tau}_{\beta_{\xi}}: \xi \in \kappa \backslash B\right\}$ before we force the $\mathcal{\tau}_{\beta}$ 's! If $\mathcal{\tau}_{\alpha}$ is similar enough to being Cohen over $\left\langle\mathcal{\tau}_{\beta_{\xi}}: \xi \in \kappa \backslash B\right\rangle$, we are done. So this becomes similar to the problem in proving 0.1. But there we use $2^{\kappa}=\chi$, so that we need to carry only $\kappa$ finitely additive measures $\left\langle\Xi_{\sim}^{t}: t \in \mathcal{T}\right\rangle$, i.e. have few blueprints, hence can make $A_{\alpha}$ (when $\left|\mathbb{Q}_{\alpha}\right| \geq \kappa$ ) closed enough. However, the $\Xi_{\alpha}^{t}$ 's had been really used only to prove the existence of $p^{\oplus}$ which forces that many $p_{l}$ 's $(l<\omega)$ are in the generic set 
(see the proof of [Sh 592, Claim 3.4]). So in fact we can define the name of the finitely additive measure after we have the sequence $\left\langle p_{l}: l<\omega\right\rangle$. Actually, we need this only for some specific cases and/or can embed our $\mathbb{P}_{\alpha}$ into another iteration. So the problem boils down to having the $A_{\beta}$ 's closed enough to enable us to produce finitely additive measures like the one in [Sh 592, 2.11(i) or 2.16(d)]. The way we materialize the idea is by having enough automorphisms of the structure

$$
\left\langle\mathbb{P}_{\alpha}, \mathbb{Q}_{\beta}, A_{\beta}, \mu_{\beta}, \tau_{\beta}: \alpha \leq \alpha^{*}, \beta<\alpha^{*}\right\rangle .
$$

In fact, below we can replace $\lambda+\kappa$ by $\lambda \cdot \kappa$. If we would like to have e.g. $\{A: \Xi(A)=1\}$ to be a selective filter then the $\lambda \times \kappa$ version is better: we can use $\left\{\tau_{\lambda \times \xi+1+\gamma}: \gamma<\lambda\right\}$, which are Cohen, to ensure this.

If you do not like the use of "automorphisms" of $\overline{\mathbb{Q}}$ and doing it through higher $\lambda$ 's, later we analyze the partial support iteration (as in [Sh 592, $\S \S 2$, 3]) more fully (using essentially $\prec_{\mathbb{L}_{\aleph_{1}, \aleph_{1}}}$ ), and then the proof is more direct (see 3.1-3.13).

1.1. TheOREM. Let $D$ be a $\kappa$-complete non-principal ultrafilter on $\kappa$. Then for some c.c.c. forcing notion $\mathbb{P}$ of cardinality $2^{\kappa}$, in $\mathbf{V}^{\mathbb{P}}$ we have:

(*) for some $A \in\left[{ }^{\omega} 2\right]^{\kappa}$ we have:

(a) $A$ is not null,

(b) the ideal $I=\{B \subseteq A: B$ is null $\}$ is $\aleph_{1}$-saturated.

Moreover,

(**) we can find pairwise distinct $\eta_{\xi} \in{ }^{\omega} 2($ for $\xi<\kappa)$ such that

$$
A=\left\{\eta_{\xi}: \xi<\kappa\right\} \quad \text { and } \quad Y \in D \Leftrightarrow\left\{\eta_{\xi}: \xi \in \kappa \backslash Y\right\} \text { is null. }
$$

1.2. Remark. (1) We can replace $\omega_{2}$ by $\mathbb{R}$.

(2) We can use as $D$ any uniform $\aleph_{2}$-complete filter on $\kappa$ (such that $D$ is $\aleph_{1}$-saturated in $\mathbf{V}$ and hence in $\mathbf{V}^{\mathbb{P}}$, see 1.12).

(3) In $(* *)$ of course $D$ stands for the filter that $D$ generated in $\mathbf{V}^{\mathbb{P}}$.

1.3. Convention. For $\lambda \geq 2^{\kappa}$ (but we use only $\lambda<\left(2^{\kappa}\right)^{+\omega}$ ) let $g_{\lambda}$ : $\lambda \rightarrow D$ be such that

$$
Y \in D \Rightarrow\left|\left\{\alpha<\lambda: g_{\lambda}(\alpha)=Y\right\}\right|=\lambda .
$$

For simplicity $g_{\lambda}$ is increasing with $\lambda$, and let $h: D \rightarrow 2^{\kappa}$ be such that $g_{\lambda} \circ h=\operatorname{id}_{D}$. For $\xi<\kappa$ let

$$
E_{\xi}=E_{\xi}^{\lambda}=\left\{\alpha<\lambda: \xi \in g_{\lambda}(\alpha)\right\} .
$$

Finally we let

$$
\bar{E}=\bar{E}^{\lambda}=\left\langle E_{\xi}^{\lambda}: \xi<\kappa\right\rangle .
$$

If not said otherwise we assume that $\lambda=\lambda^{\aleph_{0}} \geq 2^{\kappa}$ (for simplicity). 
REMARK. Our intention is that $\tau_{\alpha}$ will exemplify $\left\{\tau_{\lambda+\xi}: \xi \in \kappa \backslash g_{\lambda}(\alpha)\right\}$ is null, for which it is enough that $\tau_{\alpha}$ will be (at least somewhat) like Cohen over $\mathbf{V}\left[\left\langle\tau_{\lambda+\xi}: \xi \in \kappa \backslash g_{\lambda}(\alpha)\right\rangle\right]$, so it is reasonable to ask that $\tau_{\alpha}$ is not in the subuniverse over which $\tau_{\lambda+\xi}$ is random when $\xi \in \kappa \backslash g_{\lambda}(\alpha)$, i.e. $\alpha \notin E_{\xi}$.

1.4. Definition. (1) $\mathcal{K}_{\theta}^{\prime}$ is the family of $\overline{\mathbb{Q}}=\left\langle\mathbb{P}_{\alpha}, \mathbb{Q}_{\alpha}, A_{\alpha}, \mu_{\alpha}, \tau_{\alpha}: \alpha<\beta\right\rangle$ such that:

(A) $\left\langle\mathbb{P}_{\alpha}, \mathbb{Q}_{\alpha}: \alpha<\beta\right\rangle$ is a FS iteration.

(B) $A_{\alpha} \subseteq \alpha, \mu_{\alpha}=\aleph_{0}$, and either $\left|A_{\alpha}\right|<\theta, \mathbb{Q}_{\alpha}$ is Cohen forcing, $\tau_{\alpha}$ the Cohen real or $\left|A_{\alpha}\right| \geq \theta, \mathbb{Q}_{\alpha}$ is Random $\mathbf{V}\left[\left\langle\tau_{\gamma}: \gamma \in A_{\alpha}\right\rangle\right]$ (defined as in $[$ Sh $592,2.2])$ and $\tau_{\alpha}$ the random real. This is a particular case of [Sh 592, 2.2] except replacing $\kappa$ there by $\theta$ here, so we can use [Sh 592].

(2) We define $\mathbb{P}_{\beta}$ naturally as the direct limit of the FS iteration $\left\langle\mathbb{P}_{\alpha}, \mathbb{Q}_{\alpha}\right.$ : $\alpha<\beta\rangle$ and for $\alpha \leq \beta$ we define $\mathbb{P}_{\alpha}^{\prime}=\left\{p \in \mathbb{P}_{\alpha}\right.$ : for every $\gamma \in \operatorname{dom}(p), \widetilde{p}(\gamma)$ has the form $\mathcal{B}\left(\ldots, \text { truth value }\left(\zeta_{n} \in \tau_{\gamma_{n}}\right), \ldots\right)_{n<\omega}$ where $\mathcal{B}$ is a Borel function and $\gamma_{n} \in A_{\gamma}$ and $\left.\zeta_{n}<\omega\right\}$.

(3) We may write $\mathbb{P}_{\alpha}=\mathbb{P}_{\alpha}^{\overline{\mathbb{Q}}}$, etc.

1.5. Remark. Note that Definition 1.6(1) and Claim 1.7(2) below are vacuous when $\theta=1$, the case we use here, but they are natural for less specific cases. Let $\overline{\mathcal{\tau}}=\left\langle\tau_{\alpha}: \alpha<\beta\right\rangle$; note that more accurately we should write not $\mathbb{Q}_{\alpha}=$ Random $\mathbf{V}\left[\bar{\tau} \mid A_{\alpha}\right]$ but Random $\mathbf{V}\left[\bar{\tau}\left\lceil A_{\alpha}\right], \bar{\tau} \mid A_{\alpha}\right.$ or Random $\mathbf{V}, \bar{\tau} \mid A_{\alpha}$ as a priori $\widetilde{\mathbb{Q}}_{\alpha}$ may be a proper subset of Random $\mathbf{V}\left[\bar{\tau} \mid A_{\alpha}\right]$ depending on $\overline{\mathcal{\tau}} \mid A_{\alpha}$, too. For the "good" cases equality holds, in particular in $\S 1+\S 2$ here. For brevity we shall write Random $\bar{\tau}^{\bar{\tau}} \mid A$.

1.6. Definition. For $\overline{\mathbb{Q}} \in \mathcal{K}_{\theta}^{\prime}$ :

(1) $A \subseteq \lg (\overline{\mathbb{Q}})$ is called $\overline{\mathbb{Q}}$-closed if

$$
\left(\alpha<\lg (\overline{\mathbb{Q}}) \&\left|A_{\alpha}\right|<\theta \& \alpha \in A\right) \Rightarrow A_{\alpha} \subseteq A .
$$

(2) Let $\left({ }^{1}\right)$

$\operatorname{PAUT}(\overline{\mathbb{Q}})=\{f: f$ is a one-to-one partial function from $\lg (\overline{\mathbb{Q}})$ to $\lg (\overline{\mathbb{Q}})$ such that $\operatorname{dom}(f)$ and $\operatorname{rang}(f)$ are $\overline{\mathbb{Q}}$-closed and for $\beta \in \operatorname{dom}(f)$ we have $\left|A_{\beta}\right|<\theta \Leftrightarrow\left|A_{f(\beta)}\right|<\theta$ and if $\beta, \gamma \in \operatorname{dom}(f)$, then $\left.\beta \in A_{\gamma} \Leftrightarrow f(\beta) \in A_{f(\gamma)}\right\}$.

(3) We define the following by induction on $\lg (\overline{\mathbb{Q}})$. For $f \in \operatorname{PAUT}(\overline{\mathbb{Q}})$ let $\widehat{f}$ be the partial function from $\mathbb{P}_{\operatorname{dom}(f)}^{\prime}$ to $\mathbb{P}_{\text {rang }(f)}^{\prime}$ (see [Sh 592, Definition

$\left.{ }^{1}\right)$ This suffices when we iterate just partial random forcing (when $\left|A_{\beta}\right| \geq \kappa$ ) and Cohen forcing (when $\left|\mathbb{Q}_{\beta}\right|<\kappa$ ), which is enough here. For a more complicated situation, see $\S 3$. 
2.2(3)]) defined by

$$
\begin{array}{r}
p_{1}=\widehat{f}\left(p_{0}\right) \quad \text { when } p_{0} \in \mathbb{P}_{\operatorname{dom}(f)}^{\prime}, \quad \operatorname{dom}\left(p_{1}\right)=\left\{f(\beta): \beta \in \operatorname{dom}\left(p_{0}\right)\right\}, \\
p_{1}(f(\beta))=\mathcal{B}_{\beta}\left(\ldots, \operatorname{truth} \operatorname{value}\left(\zeta \in \mathcal{I}_{f(\gamma)}\right), \ldots\right)_{\gamma \in w_{\beta}^{p_{0}}, \zeta<\omega} \\
\text { when } p_{0}(\beta)=\mathcal{B}_{\beta}\left(\ldots, \operatorname{truth} \operatorname{value}\left(\zeta \in \mathcal{I}_{\gamma}\right), \ldots\right)_{\gamma \in w_{\beta}^{p_{0}}, \zeta<\omega}
\end{array}
$$

$\left(\right.$ so $\left.w_{f(\beta)}^{p_{1}}=\left\{f(\gamma): \gamma \in w_{\beta}^{p_{0}}\right\}\right)$.

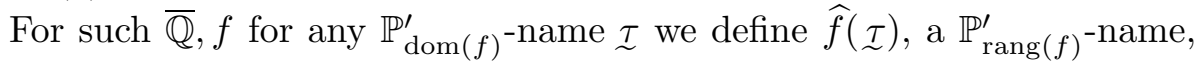
naturally (when we define names without using the order or being a (maximal) antichain).

(4) For $\overline{\mathbb{Q}} \in \mathcal{K}_{\theta}^{\prime}$ let

$$
\operatorname{EAUT}(\overline{\mathbb{Q}})=\{f \in \operatorname{PAUT}(\overline{\mathbb{Q}}): \operatorname{dom}(f)=\operatorname{rang}(f)=\lg (\overline{\mathbb{Q}})\} .
$$

(5) If $A$ is $\overline{\mathbb{Q}}$-closed let $\mathbb{P}_{A}^{\prime}$ be

$\left\{p \in \mathbb{P}_{\alpha}\right.$ : for each $\beta \in \operatorname{dom}(p)$ we have $\beta \in A$ and the condition $p(\beta)$ is either a Cohen condition or has the form $\mathcal{B}\left(\ldots, \text { truth value }\left(\zeta \in \mathcal{I}_{\gamma}\right), \ldots\right)_{\gamma \in w_{\beta}^{p}, \zeta<\omega}$, where $w_{\beta}^{p} \subseteq A_{\beta} \cap A$ is a countable set, $\mathcal{B}$ is a Borel function with domain and range of the right form (and $\mathcal{B}, w_{\beta}^{p}$ are not $\mathbb{P}_{\beta}$-names but actual objects)\}.

1.7. FACT. Let $\overline{\mathbb{Q}} \in \mathcal{K}_{\theta}^{\prime}$.

(1) If $\alpha<\lg (\overline{\mathbb{Q}}), \overline{\mathbb{Q}}^{\prime}=\overline{\mathbb{Q}}\left\lceil\alpha\right.$, then $\overline{\mathbb{Q}}^{\prime} \in \mathcal{K}_{\theta}^{\prime}$ and $\operatorname{PAUT}\left(\overline{\mathbb{Q}^{\prime}}\right) \subseteq \operatorname{PAUT}(\overline{\mathbb{Q}})$.

(2) If $\alpha \leq \lg (\overline{\mathbb{Q}})$, then $\{\beta: \beta<\alpha\}$ is $\overline{\mathbb{Q}}$-closed; and the family of $\overline{\mathbb{Q}}$-closed sets is closed under intersection and union (of any family).

(3) If $f \in \operatorname{PAUT}(\overline{\mathbb{Q}})$ and $A \subseteq \lg (\overline{\mathbb{Q}})$ is $\overline{\mathbb{Q}}$-closed, then $f \uparrow(A \cap \operatorname{dom}(f))$ belongs to $\operatorname{PAUT}(\overline{\mathbb{Q}})$.

(4) If $\left(^{2}\right) f \in \operatorname{EAUT}(\overline{\mathbb{Q}})$, then $\widehat{f}$ is an automorphism of $\mathbb{P}_{\lg (\overline{\mathbb{Q}})}^{\prime}$.

(5) If $A$ is $\overline{\mathbb{Q}}$-closed and $\otimes_{\overline{\mathbb{Q}}, A}$ below holds, then $\mathbb{P}_{A}^{\prime} \lessdot \mathbb{P}_{\lg (\overline{\mathbb{Q}})}$, where $\otimes_{\overline{\mathbb{Q}}, A} \quad$ if $\alpha \in A,\left|A_{\alpha}\right| \geq \theta$ and $B \subseteq \alpha$ is countable, then for some $f \in$ $\operatorname{EAUT}(\overline{\mathbb{Q}} \mid \alpha)$ we have:

(i) $f \uparrow(B \cap A)=$ the identity,

(ii) $f^{\prime \prime}(B) \subseteq A$,

(iii) $f^{\prime \prime}\left(B \cap A_{\alpha}\right) \subseteq A \cap A_{\alpha}$.

Proof. Straightforward. For part (5) we prove by induction on $\beta \leq l(\overline{\mathbb{Q}})$ that if we replace $\mathbb{P}_{\lg (\overline{\mathbb{Q}})}^{\prime}, A$ by $\mathbb{P}_{\beta}^{\prime}, A^{\prime}=A \cap \beta$, so $q \in \mathbb{P}_{\beta}^{\prime}$, then the conclusion of (5) holds.

$\left(^{2}\right)$ Does $f \in \operatorname{PAUT}(\overline{\mathbb{Q}})$ imply $\widehat{f}$ is an isomorphism from $\mathbb{P}_{\operatorname{dom}(f)}^{\prime}$ onto $\mathbb{P}_{\operatorname{rang}(f)}^{\prime}$ ? The problem is that the order is inherited from $\mathbb{P}_{\alpha}$ so in general is not necessarily the same. 
The case $\beta=0$ is trivial and the limit cases use the properties of FS iterations. At successor stages $\beta=\alpha+1$, if $\alpha \notin A$ or $\left|A_{\alpha}\right|<\theta$ it is trivial, so assume $\alpha \in A \&\left|A_{\alpha}\right| \geq \theta$. Hence by the induction hypothesis $\mathbb{P}_{A \cap \alpha}^{\prime} \lessdot \mathbb{P}_{\alpha}$ etc., and it is enough to show:

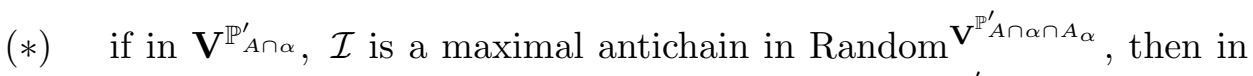
$\mathbf{V}^{\mathbb{P}_{\alpha}^{\prime}}$, the set $\mathcal{I}$ is a maximal antichain of Random $\mathbf{V}^{\mathbb{P}^{\prime} A_{\alpha}}$.

By the c.c.c. this is equivalent to

$(*)^{\prime} \quad$ if $\zeta^{*}<\omega_{1},\left\{p_{\zeta}: \zeta<\zeta^{*}\right\} \subseteq \mathbb{P}_{A \cap(\alpha+1)}^{\prime}, p \in \mathbb{P}_{A \cap \alpha}^{\prime}$ and $p \Vdash_{\mathbb{P}_{A \cap \alpha}^{\prime}}$ " $\left\{p_{\zeta}(\alpha): \zeta<\zeta^{*}\right.$ and $p_{\zeta}\left\lceil\alpha \in G_{\mathbb{P}_{A \cap \alpha}^{\prime}}\right\}$ is a predense subset of

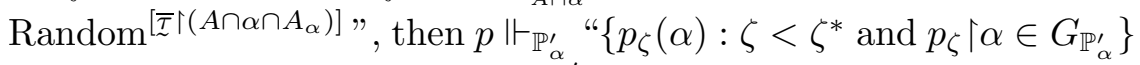
is a predense subset of Random $\mathbf{V}^{\mathbb{P}^{\prime} A_{\alpha}}$ ".

Assume $(*)^{\prime}$ fails, so we can find $q$ such that $p \leq q \in \mathbb{P}_{\alpha}^{\prime}$ and $q \Vdash_{\mathbb{P}_{\alpha}^{\prime}}$ " $\left\{p_{\zeta}(\alpha): \zeta<\zeta^{*}\right.$ and $p_{\zeta}\left\lceil\alpha \in G_{\mathbb{P}_{\alpha}^{\prime}}\right\}$ is not a predense subset of Random $\overline{\mathcal{\tau}}^{\lceil} A_{\alpha} "$.

So for some $\mathbb{P}_{\alpha}^{\prime}$-name $\underset{\sim}{r}$ we have

$$
\begin{aligned}
q \Vdash_{\mathbb{P}_{\alpha}^{\prime}} \text { " } r \in \operatorname{Random}^{\bar{\tau} \mid A_{\alpha}}(=\underset{\mathbb{Q}}{\alpha}) \text { and } & \\
& \text { it is incompatible with every } p_{\zeta}(\alpha) \in \mathbb{Q}_{\alpha} " .
\end{aligned}
$$

Possibly increasing $q$, without loss of generality $\underset{\sim}{r}$ is

$$
\mathcal{B}\left(\ldots, \text { truth value }\left(\zeta \in \tau_{\gamma}\right), \ldots\right)_{\gamma \in w, \zeta<\omega},
$$

where $w \subseteq A_{\alpha}$ is countable. Let us define (supp for support is from [Sh 592, Def. $2.2(1)(\mathrm{F})$ ], i.e. above $\operatorname{supp}(\underset{\sim}{r})=w)$

$$
\begin{aligned}
B= & w \cup \operatorname{dom}(q) \cup \bigcup_{\zeta<\zeta^{*}} \operatorname{dom}\left(p_{\zeta}\lceil\alpha) \cup \bigcup\{\operatorname{supp}(q(\beta)): \beta \in \operatorname{dom}(q)\}\right. \\
& \cup \bigcup\left\{\operatorname{supp}\left(p_{\zeta}(\beta)\right): \beta \in \operatorname{dom}\left(p_{\zeta}\lceil\alpha) \text { and } \zeta<\zeta^{*}\right\} .\right.
\end{aligned}
$$

Clearly $B$ is a countable subset of $\alpha$. By $\otimes_{\overline{\mathbb{Q}}, A}$ there is $f \in \operatorname{EAUT}(\overline{\mathbb{Q}}\lceil\alpha)$ such that

$$
f \uparrow(B \cap A)=\text { the identity, } \quad f^{\prime \prime}(B) \subseteq A, \quad f^{\prime \prime}\left(B \cap A_{\alpha}\right) \subseteq A_{\alpha} .
$$

As $\widehat{f}$ is an automorphism of $\mathbb{P}_{\alpha}^{\prime}$ and is the identity on $\mathbb{P}_{A \cap B}^{\prime}$, clearly

$$
\widehat{f}(p)=p, \quad \widehat{f}\left(p_{\zeta}\right)=p_{\zeta}, \quad p \leq \widehat{f}(q) \in \mathbb{P}_{\alpha}^{\prime},
$$

and $\widehat{f}(\underset{\sim}{r})$ is $\mathcal{B}\left(\ldots, \text { truth value }\left(\zeta \in \tau_{f(\gamma)}\right), \ldots\right)_{\gamma \in w, \zeta<\omega}$, and

$$
f^{\prime \prime}(w) \subseteq f^{\prime \prime}\left(B \cap A_{\alpha}\right) \subseteq A \cap A_{\alpha} .
$$

Hence $\Vdash_{\mathbb{P}_{\alpha}^{\prime}}$ " $f(\underset{\sim}{r}) \in$ Random $\mathcal{\mathcal { T }}^{\bar{\tau}\left(A \cap A_{\alpha}\right)}$ " and 
$\widehat{f}(q) \Vdash_{\mathbb{P}_{A}^{\prime}}$ "in $\underset{\sim}{\mathbb{Q}} \alpha$, the conditions $f(\underset{\sim}{r})$ and $p_{\zeta}(\alpha)$

are incompatible for $\zeta<\zeta^{*}$,

so we get a contradiction.

Note that we use: in $\mathbf{V}^{\mathbb{P}_{\alpha}^{\prime}}$, two conditions in Random $\bar{\tau} \mid B$ are compatible in Random $\tilde{\tau}^{\bar{\tau} \mid B}$ iff they are compatible in Random $\mathbf{V}^{\mathbb{P}_{\alpha}^{\prime}}$, for every $B \subseteq \alpha$; in particular for $B=A_{\alpha} \cap A$ and $B=f^{\prime \prime}\left(A_{\alpha} \cap A\right)$. $\mathbf{m}_{1.7}$

1.8. REMARK. (1) Instead of automorphisms $(f \in \operatorname{EAUT}(\overline{\mathbb{Q}}\lceil\alpha))$ we can use $f \in \operatorname{PAUT}(\overline{\mathbb{Q}}\lceil\alpha)$ but having more explicit assumptions and more to carry by induction; see $\S 3$.

(2) The use of Random is not essential for the last claim, we just need enough absoluteness.

Proof of 1.1. We shall define

$$
\overline{\mathbb{Q}}^{\lambda}=\left\langle\mathbb{P}_{\alpha},{\underset{\mathbb{Q}}{\beta}}_{\beta}, A_{\beta}, \mu_{\beta}, \tau_{\beta}: \alpha \leq \lambda+\kappa \text { and } \beta<\lambda+\kappa\right\rangle
$$

as an iteration from $\mathcal{K}_{\theta}^{\prime}$. More accurately, $\mathbb{P}_{\alpha}=\mathbb{P}_{\alpha}^{\lambda}=\mathbb{P}_{\lambda, \alpha}, \mathbb{Q}_{\alpha}=\mathbb{Q}_{\alpha}^{\lambda}=\mathbb{Q}_{\lambda, \alpha}$, $A_{\alpha}=A_{\alpha}^{\lambda}=A_{\lambda, \alpha}, \mu=\mu_{\alpha}=\mu_{\alpha}^{\lambda}, \tau_{\alpha}=\tau_{\alpha}^{\lambda}=\tau_{\lambda, \alpha}$ are suç that for $\widetilde{\beta}<\lambda$ we have: $A_{\beta}=\emptyset$ and $\mathbb{Q}_{\beta}$ is as in [Sh 592, Def. 2.5], i.e. the Cohen forcing notion (and $\tau_{\beta}$ is the Cohen real), while for $\beta \in[\lambda, \lambda+\kappa$ ) we let

$$
A_{\beta}=E_{\beta-\lambda}^{\lambda} \cup[\lambda, \beta), \quad{\underset{\mathbb{Q}}{\beta}}_{\beta}=\operatorname{Random}^{\bar{\tau} \mid A_{\beta}},
$$

and $\tau_{\beta}$ is the (partial) random real (as in [Sh 592, Definition 2.2(F)]).

Let $\mathbb{P}=\mathbb{P}^{\lambda}=\mathbb{P}_{\lambda+\kappa}$. We define $\mathbb{P}_{\alpha}^{\prime}\left(=\mathbb{P}_{\lambda, \alpha}^{\prime}\right)$ as in 1.6(5) (which is [Sh 592, Definition 2.2(3)]). More generally, $\mathbb{P}_{\lambda, A}^{\prime}$ for $A \subseteq \lambda+\kappa$ denotes $\mathbb{P}_{A}^{\prime}$ for $\overline{\mathbb{Q}}^{\lambda}$ (this is to help when we deal with more than one $\lambda$ ).

1.9. Definition/Fact. (1) We define $\mathcal{E}_{\xi}^{\lambda}$ (for $\xi<\kappa$ and $\lambda=\lambda^{\aleph_{0}} \geq 2^{\kappa}$ ), an equivalence relation on $\lambda$ by: $\alpha \mathcal{E}_{\xi}^{\lambda} \beta$ iff $g_{\lambda}(\alpha) \cap \xi=g_{\lambda}(\beta) \cap \xi$.

(2) $\mathcal{E}_{\xi}^{\lambda}$ is an equivalence relation on $\lambda$ with $\leq 2^{|\xi|}$ equivalence classes, each of cardinality $\lambda$.

(3) If $\xi \leq \zeta<\kappa$, then $\mathcal{E}_{\zeta}^{\lambda}$ refines $\mathcal{E}_{\xi}^{\lambda}$ and $\left|E_{\zeta}^{\lambda} \cap\left(\alpha / \mathcal{E}_{\xi}^{\lambda}\right)\right|=\lambda$ for every $\alpha<\lambda$.

1.10. FACT. (1) If $\xi<\kappa$, then $\left(\right.$ in $\left.\overline{\mathbb{Q}}^{\lambda}\right)$ :

(a) $\mathbb{P}_{A_{\lambda+\xi}}^{\prime}=\mathbb{P}_{\left(\lambda \cap A_{\lambda+\xi} \cup[\lambda, \lambda+\xi)\right.}^{\prime}=\mathbb{P}_{E_{\xi}^{\lambda} \cup[\lambda, \lambda+\xi)}^{\prime} \lessdot \mathbb{P}_{\lambda+\xi}^{\prime}$.

(b) If $q \in \mathbb{P}_{\lambda+\xi}^{\prime}$ and $q \uparrow\left(E_{\xi}^{\lambda} \cup[\lambda, \lambda+\xi)\right) \leq p \in \mathbb{P}_{E_{\xi}^{\lambda} \cup[\lambda, \lambda+\xi)}^{\prime}$, then

$$
p \cup\left(q \uparrow\left(\lambda \backslash E_{\xi}^{\lambda}\right) \cup[\lambda, \lambda+\xi)\right) \in \mathbb{P}_{\lambda+\xi}^{\prime}
$$

is the least upper bound of $p, q$.

(2) If $\lambda \leq \lambda^{\prime}$, then $\mathbb{P}_{\lambda^{\prime}, \lambda \cup\left[\lambda^{\prime}, \lambda^{\prime}+\kappa\right)}^{\prime} \lessdot \mathbb{P}_{\lambda^{\prime}, \lambda^{\prime}+\kappa}^{\prime}$ and $\mathbb{P}_{\lambda^{\prime}, \lambda \cup\left[\lambda^{\prime}, \lambda^{\prime}+\kappa\right)}^{\prime}$ is isomorphic to $\mathbb{P}_{\lambda, \lambda+\kappa}^{\prime}$, say by $h: \mathbb{P}_{\lambda, \lambda+\kappa}^{\prime} \rightarrow \mathbb{P}_{\lambda^{\prime}, \lambda \cup\left[\lambda^{\prime}, \lambda^{\prime}+\kappa\right)}^{\prime}$, where $h=h^{\lambda, \lambda^{\prime}}$ is 
the canonical mapping, i.e. let $h: \lambda+\kappa \rightarrow \lambda^{\prime}+\kappa$ be $\alpha<\lambda \Rightarrow h(\alpha)=\alpha$ and $\xi<\kappa \Rightarrow h(\lambda+\xi)=\lambda^{\prime}+\xi$, now if $h(p)=p^{\prime}$ then $\operatorname{dom}(h(p))=\{h(\alpha): \alpha \in$ $\operatorname{dom}(p)\}$, etc.

(3) If $\alpha<\lambda+\kappa$ and $\left|A_{\alpha}\right| \geq \theta$ (equivalently, $\alpha \in[\lambda, \lambda+\kappa)$ ), then $\underset{\sim}{\mathbb{Q}_{\lambda, \alpha} \in \mathbf{V}^{\bar{\tau}} \mid A_{\alpha}}=\mathbf{V}^{\mathbb{P}_{A_{\alpha}}^{\prime}}$.

Proof. (1) By 1.7(5) (really this is a particularly simple case). That is, let $A=A_{\lambda+\xi}=E_{\xi}^{\lambda} \cup[\lambda, \lambda+\xi)$, so by $1.7(5)$ it is enough to check $\otimes_{\mathbb{Q} \vdash}(\lambda+\xi) \backslash A$ there. So let $\alpha \in A$ be such that $\left|A_{\alpha}\right| \geq \theta$ and $B \subseteq \alpha$ is countable, and we should find $f$ as there. As $\left|A_{\alpha}\right| \geq \theta$, necessarily $\alpha \geq \lambda$, so $\alpha=\lambda+\zeta$ for some $\zeta<\xi$. By 1.9(3) the existence of $f$ is immediate.

(2) Straightforward.

(3) By [Sh 592, 2.3(7)] and clause (a) of part (1). $\mathbf{- 1 . 1 0}$

1.11. FACT. $X^{*}=\left\{\tau_{\lambda+\xi}: \xi<\kappa\right\}$ is not null (in $\left.\mathbf{V}^{\mathbb{P}_{\lambda+\kappa}}\right)$.

Proof. If it is null, it is included in a Borel null set $X$ which is coded by a real $s$ which is determined by $\left\langle\right.$ truth value $\left.\left(p_{l} \in G_{\mathbb{P}_{\lambda+\kappa}}\right): l<\omega\right\rangle$ for some $\left\langle p_{l}: l<\omega\right\rangle$, where $p_{l} \in \mathbb{P}_{\lambda+\kappa}^{\prime}$. Let

$$
w=\bigcup_{l<\omega} \operatorname{dom}\left(p_{l}\right) \cup \bigcup\left\{\operatorname{supp}\left(p_{l}(\alpha)\right) \text { : for some } l<\omega, \alpha \in \operatorname{dom}\left(p_{l}\right)\right\},
$$

so $w \in[\lambda+\kappa] \leq \aleph_{0}$ and $w \cap \lambda \in[\lambda] \leq \aleph_{0}$. Now

$\otimes \quad$ there is $\xi<\kappa$ such that $(\xi>0$ and):

(i) $(\forall \gamma)(\lambda+\gamma \in w \Rightarrow \gamma<\xi)$.

(ii) $\alpha \in w \cap \lambda \Rightarrow \alpha \in E_{\xi}^{\lambda}\left(\subseteq A_{\lambda+\xi}\right)$.

This is possible as we have $\aleph_{0}$ demands; for each of them the set of $\xi<\kappa$ satisfying it is in $D$. Now $\tau_{\lambda+\xi}$ is random over $\mathbf{V}^{\mathbb{P}_{A_{\lambda+\xi}}^{\prime}}$, and $X$ is (definable) in $\mathbf{V}^{\mathbb{P}_{A \lambda+\xi}^{\prime}}$ (see $\left[\right.$ Sh 592, 2.3]). Hence $\tau_{\lambda+\xi}$ is not in the Borel set $X$, a contradiction. (Alternatively follow the proof of [Sh 592, 2.3(2)].) $\mathbf{m}_{1.11}$

1.12. FACT. If $D$ is a $\kappa$-complete ultrafilter, then in $\mathbf{V}^{\mathbb{P}}, D$ is a $\kappa$ complete $\aleph_{1}$-saturated filter.

Proof. Well known (see, e.g., [J]), as $\mathbb{P}$ is a c.c.c. forcing notion.

So the "only" point left is:

1.13. FACT. If $Y \in D$, then $\Vdash_{\mathbb{P}}$ " $\left\{\tau_{\lambda+\xi}: \xi \in \kappa \backslash Y\right\}$ is null".

This follows from

1.14. MAIN FACT. If $\xi<\kappa, \alpha<\lambda, \alpha \notin E_{\xi}$, then $\Vdash_{\mathbb{P}_{\lambda, \lambda+\kappa}^{\prime}}{ }{ } \tau_{\lambda+\xi} \in$ $N\left(\tau_{\alpha}\right)$ ", where $N\left(\tau_{\alpha}\right)$ is as in [Sh 592, Definition 2.4]. 
Proof. Note that

(*) for $\xi<\kappa$,

$$
\begin{aligned}
\operatorname{EAUT}(\overline{\mathbb{Q}} \uparrow(\lambda+\xi)) \supseteq\{f: & f \text { a permutation of } \lambda+\xi, \\
& f \uparrow[\lambda, \lambda+\xi) \text { is identity and } \\
& f\left\lceil\lambda \text { maps } E_{\gamma}^{\lambda} \text { onto } E_{\gamma}^{\lambda} \text { for } \gamma<\xi\right\} .
\end{aligned}
$$

Assume toward a contradiction that the desired conclusion fails, hence for some $p \in \mathbb{P}_{\lambda+\kappa}$ we have $p \Vdash$ " $\tau_{\lambda+\xi} \notin N\left(\mathcal{\tau}_{\alpha}\right)$ ". In fact, possibly increasing $p$, without loss of generality for some $l^{*}$ we have

$$
p \Vdash " \bigwedge_{l \geq l^{*}} \tau_{\lambda+\xi}\left\lceil n_{l}^{\alpha} \in a_{l}^{\alpha} ",\right.
$$

where $\left\langle n_{l}^{\alpha}, a_{l}^{\alpha}: l<\omega\right\rangle$ is as in [Sh 592, Definition 2.4].

We may assume that $p \in \mathbb{P}_{\lambda+\xi+1}$. Let $G \subseteq \mathbb{P}_{\lambda+\xi}$ be generic over $\mathbf{V}$ such that $p \uparrow(\lambda+\xi) \in G$. So in $\mathbf{V}[G]$,

$$
p(\lambda+\xi) \Vdash_{\mathbb{Q}_{\lambda+\xi}} \text { " } \tau_{\lambda+\xi} \in \underset{l_{l *}^{T}}{\alpha},
$$

where

$\underset{\sim}{T_{l^{*}}^{\alpha}}=T_{l^{*}}\left(\underline{\bar{a}}^{\alpha}\right):=\left\{\eta \in{ }^{\omega>} 2\right.$ : if $l \in\left[l^{*}, \omega\right)$ and $\underline{\sim}_{l}^{\alpha} \leq \lg (\eta)$, then $\eta\left\lceil\sim_{l}^{\alpha} \in \underline{a}_{l}^{\alpha}\right\}$.

Now for some $q$ we have $p \uparrow(\lambda+\xi) \leq q \in G$ and

$$
q \Vdash " p(\lambda+\xi)=\mathcal{B}\left(\ldots, \operatorname{truth} \text { value }\left(\varepsilon_{l} \in \mathcal{\tau}_{\beta_{l}}\right), \ldots\right)_{l<\omega},
$$

where $\mathcal{B} \in \mathbf{V}$ is a Borel function, $\beta_{l} \in A_{\lambda+\xi}$ and $\varepsilon_{l}<\mu_{\beta_{l}}$ for $l<\omega$. So (see [Sh 592, Definition 2.2(1)(F)( $\alpha)]$ )

$$
\underline{\mathbf{t}}:=\mathcal{B}\left(\ldots, \text { truth value }\left(\varepsilon_{l} \in \tau_{\beta_{l}}\right), \ldots\right)_{l<\omega}
$$

is a perfect subtree of $(\omega>2, \triangleleft)$ of positive measure above every node, i.e.

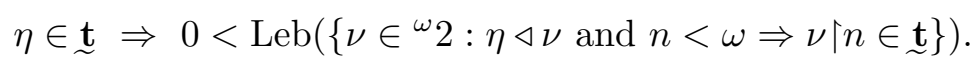

So by the choice of $p$ and $q$ we have $q \Vdash$ "t $\subseteq{\underset{\sim}{l^{*}}}^{\alpha}$ ". Without loss of generality $q \in \mathbb{P}_{\lambda+\xi}^{\prime}$. Let

$$
w^{*}=\operatorname{dom}(q) \cup \bigcup\{\operatorname{supp}(q(\zeta)): \zeta \in \operatorname{dom}(q)\} \cup\left\{\beta_{l}: l<\omega\right\} \cup\{\alpha\} .
$$

We can choose $p_{\zeta} \in \mathbb{P}_{\lambda+\xi}, f_{\zeta}, \alpha_{\zeta}$ (by induction on $\zeta<\left(2^{\aleph_{0}}\right)^{+}$) such that:

(a) $f_{\zeta} \uparrow\left(w^{*} \backslash\{\alpha\}\right)=$ the identity.

(b) $f_{\zeta} \uparrow[\lambda, \lambda+\xi+1)=$ the identity.

(c) $f_{\zeta} \in \operatorname{EAUT}(\overline{\mathbb{Q}} \uparrow(\lambda+\xi+1))$.

(d) $\alpha_{\zeta}=f_{\zeta}(\alpha)$.

(e) $\alpha_{\zeta} \notin\left\{\alpha_{\varepsilon}: \varepsilon<\zeta\right\}$.

(f) $q_{\zeta}=\widehat{f}_{\zeta}(q)$ and $\beta_{l}^{\zeta}=f_{\zeta}\left(\beta_{l}\right)$ for $l<\omega$.

Hence: 


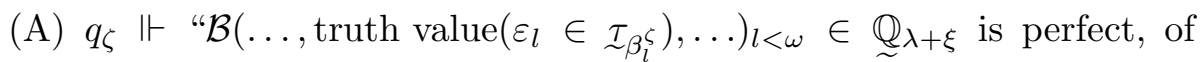
positive measure (above every node), and $\subseteq T_{l^{*}}\left(\bar{a}^{\alpha_{\zeta}}\right)$ " (also for the last statement we need " $p_{\zeta} \Vdash$ ").

(B) $\zeta_{1}<\zeta_{2} \Rightarrow \alpha_{\zeta_{1}} \neq \alpha_{\zeta_{2}}$.

(C) $\alpha_{\zeta} \notin A_{\lambda+\xi}$.

So if $G_{\mathbb{P}_{\lambda+\xi}} \subseteq \mathbb{P}_{\lambda+\xi}$ is generic over $\mathbf{V}$ and $E=\left\{\zeta<\left(2^{\aleph_{0}}\right)^{+}: q_{\zeta} \in G_{\mathbb{P}_{\lambda+\xi}}\right\}$ is unbounded, we see that in the universe $\mathbf{V}\left[G_{\mathbb{P}_{\lambda+\xi}}\right]$ for some unbounded subset $E$ of $\left(2^{\aleph_{0}}\right)^{+}$the set $Y=\bigcap_{\zeta \in E} T_{l^{*}}\left(\widetilde{a}^{\alpha}\right)\left[G_{\mathbb{P}_{\lambda+\xi}}\right]$ has an $\omega$-branch $\tau_{\lambda+\xi}$ in $\left(\mathbf{V}\left[G_{\mathbb{P}_{\lambda+\xi}}\right]\right)^{\mathbb{Q}_{\lambda+\xi}}$ which is not in $\mathbf{V}\left[G_{\mathbb{P}_{\lambda+\xi}}\right]$ (think), but this set $Y$ is a subtree of $(\omega>2, \triangleleft)$. Hence $\left({ }^{3}\right) Y$ contains a perfect subtree, which was exactly our problem in proving Theorem 0.1 in [Sh 592]. So we would like to continue as in the proof of [Sh 592, 0.1], but for this we need a suitable $\underset{\sim}{\Xi}$.

First proof. We start by repeating the proof of [Sh 592, 3.3]. As there we choose a sequence $\bar{\varepsilon}=\left\langle\varepsilon_{l}: l<\omega\right\rangle$ of positive reals satisfying $\sum_{l<\omega}{\sqrt{\varepsilon_{l}}}_{l}$ 1/10. Letting $\kappa=2^{\aleph_{0}}$, for each $\zeta<\kappa^{+}$we choose $p_{\zeta} \in \mathcal{I}_{\bar{\varepsilon}}\left(\subseteq \mathbb{P}_{\lambda+\xi}^{\prime}, \mathcal{I}_{\bar{\varepsilon}}\right.$ defined in [Sh 592, 3.1]) such that $q_{\zeta} \leq p_{\zeta}$ and let $\bar{\nu}^{\zeta}=\left\langle\nu_{\beta}^{\zeta}: \beta \in \operatorname{dom}\left(p_{\zeta}\right),\left|A_{\beta}\right| \geq \theta\right\rangle$ witness $p_{\zeta} \in \mathcal{I}_{\bar{\varepsilon}}$. Replacing $\left\langle p_{\zeta}: \zeta<\kappa^{+}\right\rangle$by a subsequence of the same length, without loss of generality the bullets $(\bullet)$ (i.e. $\otimes$ ) of the proof of [Sh 592, 3.3] hold, so we get $i^{*}, \gamma_{i}^{\zeta}$ (for $i<i^{*}$ and $\zeta<\kappa^{+}$), $v_{0}, v_{1}, z$, $\left\langle\gamma_{i}: i \in v_{0}\right\rangle, \bar{\nu}=\left\langle\nu_{i}: i<v_{1}\right\rangle, s^{*}, m^{*}$ as there.

We proceed to define $p_{\zeta}^{\prime} \geq p_{\zeta}$ as in the proof of [Sh 592,3.3] and exactly as there it suffices to prove the parallel of [Sh 592, 3.4].

The difficulty in adapting the proof of $[\mathrm{Sh} 592,3.4]$ is that it uses a blueprint defined from $\left\langle p_{\zeta}: \zeta<\omega\right\rangle$, so using the $\left\langle\eta_{\beta}: \beta<\lg (\overline{\mathbb{Q}})\right\rangle$, which does not exist here. The following two possibilities seem natural.

Possibility A. Let $\chi>\lambda^{+\omega}$ and let $\mathbb{R} \in \mathbf{V}$ be the forcing notion $\{f$ : $f$ a partial function from $\chi$ to $\{0,1\}$ with countable domain $\}$. The iterations $\overline{\mathbb{Q}}^{\lambda^{+k}}$, and the properties we are interested in, are the same in $\mathbf{V}$ and in $\mathbf{V}^{\mathbb{R}}$, so we can work in $\mathbf{V}^{\mathbb{R}}$. of ${ }^{\kappa} 2$.

So we can choose $\left\langle\underline{\eta}_{\alpha}: \alpha<\chi\right\rangle$, forced to be pairwise distinct members

PossibiLity B (Definition). The set $\mathcal{T}^{-}$of weak blueprints as in [Sh 592, Definition 2.9] replacing $w^{t}$ and the $\bar{\eta}^{t}$ 's by $\bar{\gamma}^{t}$ so replacing clauses (a), (c), (i), (j) by:
(a) ${ }^{\prime} \bar{\gamma}^{t}=\left\langle\gamma_{\mathbf{n}, k}^{t}: \mathbf{n}<\mathbf{n}^{t}, k<\omega\right\rangle$.
$(\mathrm{c})^{\prime} \gamma_{\mathbf{n}, k}^{t}$ is an ordinal increasing with $\mathbf{n}$.

$\left(^{3}\right)$ In fact, we could have demanded $\beta_{\zeta}^{l}=\beta_{l}$ for $l<\omega, \zeta<\left(2^{\aleph_{0}}\right)^{+}$, so $\mathbf{t}$ hence $Y$ includes $\mathbf{t}$ itself. 
(i) $\gamma^{\prime} \gamma_{\mathbf{n}_{1}, k_{1}}^{t}=\gamma_{\mathbf{n}_{2}, k_{2}}^{t} \Rightarrow \mathbf{n}_{1}=\mathbf{n}_{2}$.

$(\mathrm{j})^{\prime}$ For each $\mathbf{n} \leq \mathbf{n}^{t}$ the sequence $\left\langle\gamma_{\mathbf{n}, k}^{t}: k<\omega\right\rangle$ is constant or is strictly increasing; if it is constant and $\mathbf{n} \in \operatorname{dom}\left(h_{0}^{t}\right)$, then $h_{0}^{t}(n)$ is constant.

$(\mathrm{k})^{\prime}$ For $k_{2}<\mathbf{n}_{1}<\mathbf{n}_{2}<\mathbf{n}^{t}$ one of the following occurs:

( $\alpha) \mathbf{n}^{t^{*}}, \mathbf{m}^{t^{*}}, h_{0}^{t^{*}}, h_{1}^{t^{*}}, h_{2}^{t^{*}}, \bar{n}^{t^{*}}$ are as in the proof of [Sh 592,3.4].

( $\beta) \gamma_{i, \zeta}^{t^{*}}$ is $\gamma_{i, \zeta} \in \operatorname{dom}\left(p_{\zeta}^{\prime}\right)$ above (for $i<i^{*}=\mathbf{n}^{t}$ and $\zeta<\omega$ ).

Nothing relevant to us changes.

The actual difference between the two possibilities for the rest of the proof is small and we shall use the second. We define the weak blueprint $t^{*}=t(*)=\left(\bar{\gamma}^{t^{*}}, \mathbf{n}^{t^{*}}, \mathbf{m}^{t^{*}}, h_{0}^{t^{*}}, h_{1}^{t^{*}}, h_{2}^{t^{*}}, \bar{n}^{t^{*}}\right)$.

Clearly $t^{*} \in \mathcal{T}^{-}$; we would like to proceed and choose $\underset{\sim}{\Xi}$, but first choose $B_{\lambda, n} \subseteq \lambda$ for $n<\omega$ such that: if $Y_{\xi} \in\left\{A_{\lambda, \lambda+\xi} \cap \lambda, \lambda \backslash A_{\lambda, \lambda+\varepsilon}\right\}$ for $\xi<$ $\kappa, X_{n} \in\left\{B_{\lambda, n}, \lambda \backslash B_{\lambda, n}\right\}$ and $\bigcap_{\xi<\kappa} Y_{\xi} \neq \emptyset$, then $\bigcap_{\xi<\kappa} Y_{\xi} \cap \bigcap_{n<\omega} X_{n} \subseteq \lambda$ has cardinality $\lambda$ and the $B_{n}$ separates the $\gamma_{\mathbf{n}, k}^{t^{*}}$ for $\mathbf{n}<\mathbf{m}^{t^{*}}$ and $k<\omega$. Now for $\xi<\lambda$ and $\mathbf{n}<\mathbf{m}^{t(*)}$ let $\Delta_{\lambda, \xi, \mathbf{n}, k}=\{\gamma<\lambda$ : for every $\zeta<\xi$ we have $\gamma_{\mathbf{n}, k}^{t(*)} \in A_{\lambda, \lambda+\zeta} \equiv \gamma \in A_{\lambda, \lambda+\zeta}$ and $\left.\gamma_{\mathbf{n}, k}^{t^{*}} \in B_{\lambda, n} \equiv \gamma \in B_{\lambda, n}\right\}$.

For $\xi<\lambda$ let $\mathcal{T}_{\lambda, \xi}=\left\{t^{*} \in \mathcal{T}^{-}:\left(\mathbf{d}^{t(*)}, \mathbf{m}^{t(*)}, h_{0}^{t(*)}, h_{1}^{t(*)}, h_{2}^{t(*)}, \bar{n}^{t(*)}\right)=\right.$ $\left(\mathbf{n}^{t}, \mathbf{m}^{t}, h_{0}^{t}, h_{1}^{t}, h_{2}^{t}, \bar{n}^{t}\right), \mathbf{n} \in\left[\mathbf{m}^{t}, \mathbf{n}^{t}\right) \& k<\omega \Rightarrow \gamma_{\mathbf{n}, k}^{t(*)}=\gamma_{\mathbf{n}, k}^{t}$ and $\mathbf{n}<\mathbf{m}^{t} \Rightarrow$ $\left.\gamma_{n, k}^{t} \in \Delta_{\lambda, \xi, \mathbf{n}, k}\right\}$.

Let

$$
\Gamma=\left\{\gamma_{\mathbf{n}, 0}^{t(*)}, \gamma_{\mathbf{n}, 0}^{t}+1: \gamma_{\mathbf{n}, 0}^{t^{*}}=\gamma_{\mathbf{n}, 1}^{t}\right\} \cup\{\lambda\} \cup\left\{\bigcup_{k<w} \gamma_{\mathbf{n}, k}^{t^{*}}: \gamma_{n, 0}^{t}<\gamma_{n, k}^{t^{*}}\right\}
$$

for any (equivalently some) $t \in \mathcal{T}_{\lambda, 0}$; and let $\left\{\gamma_{n}^{j}: n \leq n(*)\right\}$ list $\Gamma \backslash \lambda$ in increasing order.

Now we choose the sequence $\langle\underset{\Xi}{\Xi}: j<\omega\rangle$ by induction on $n \leq n(*)$ similarly to [Sh 592, Definition 2.11] but just for the $t \in \mathcal{T}_{\lambda^{+j}, \gamma_{n}^{j}}$ :

(a) $\Xi_{n}^{j}$ is a $\mathbb{P}_{\lambda^{+j}, \gamma_{n}^{j}}$-name of a full finitely additive measure (on $\mathcal{P}(\omega)$ ).

(b) If $\mathbf{n}<\mathbf{n}^{t^{*}}, \gamma_{\mathbf{n}, k}^{j}<\gamma_{n}^{j}$ for every $k<\omega$ and $\gamma_{\mathbf{n}, 0}^{t^{*}}<\gamma_{\mathbf{n}, 1}^{t^{*}}$ and $t \in \mathcal{T}_{\lambda^{+j}, \gamma_{n}^{j}}$, then $\Vdash_{\mathbb{P}_{\lambda+j, \gamma_{n}^{j}}}$ "the following set has $\underset{\sim}{\Xi} n^{j}$-measure 1: $\left\{k<\omega:\right.$ if $l \in\left[n_{k}^{t}, n_{k+1}^{t}\right)$, then $\left.\left(h_{0}^{t}(\mathbf{n})\right)(l) \in G_{\gamma_{\mathbf{n}, l}^{j}}\right\} "$.

(c) If

$(\alpha) t \in T_{\lambda^{+j}, \gamma_{n}^{j}}, \mathbf{n}<\mathbf{n}^{t}, \gamma_{\mathbf{n}, k}^{j}<\gamma_{n}^{j}$ for $k<\omega$ and $\gamma_{\mathbf{n}, 0}^{j}=\gamma_{\mathbf{n}, 1}^{j}, n \in$ $\operatorname{dom}\left(h_{1}^{t}\right)$,

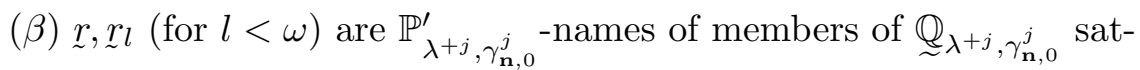
isfying $(* *)_{\sim},\langle\underset{\sim}{r}: l<\omega\rangle$ below, 
then

$\Vdash_{\mathbb{P}_{\lambda+j, \gamma_{n}^{j}}}$ "if $\underset{\sim}{r} \in{\underset{\sim}{\gamma_{n}^{j}}}$, then $1-h_{1}^{t}(\mathbf{n})$

$$
\leq \operatorname{Av}_{\underset{\Xi_{n}^{j}}{j}}\left(\left\langle\left|\left\{l \in\left[n_{k}^{t}, n_{k+1}^{t}\right):{\underset{\sim}{r} l}_{l} \in G_{\mathbb{Q}_{\gamma_{n}^{j}}}\right\}\right| /\left(n_{k+1}^{t}-n_{k}^{t}\right): k<\omega\right\rangle\right) "
$$

where

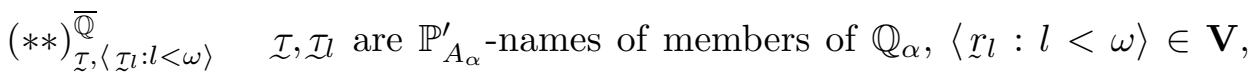
and in $\mathbf{V}^{\mathbb{P}_{\alpha}}$, for every $\tau^{l} \in \mathbb{Q}_{\alpha}$ satisfying $\tau \leq \tau^{\prime}$ we have $\operatorname{Av}_{\Xi_{\alpha}^{\tau}}\left(\left\langle a_{k}\left(\tau^{\prime}\right): k<\omega\right\rangle\right) \geq 1-h_{1}^{t}(n)$,

where

$\bigotimes$

$$
\begin{aligned}
\otimes a_{k}\left(\tau^{\prime}\right) & =a_{k}\left(\tau^{\prime}, \bar{\tau}\right)=a_{k}\left(\tau^{\prime}, \bar{\tau}, \bar{n}^{t}\right) \\
& =\left(\sum_{l \in\left[n_{k}^{t}, n_{k+1}^{t}\right)} \frac{\operatorname{Leb}\left(\lim \left(r^{\prime}\right) \cap \lim \left(r_{l}\right)\right)}{\operatorname{Leb}\left(\lim \left(r^{\prime}\right)\right)}\right) \frac{1}{n_{k+1}^{t}-n_{k}^{t}}
\end{aligned}
$$

(so $a_{k}\left(r^{\prime}, \bar{r}, \bar{n}\right) \in[0,1]$ is well defined for $k<\omega, \bar{r}=\left\langle r_{l}: l<\omega\right\rangle,\left\{r, r_{l}\right\} \subseteq$ Random, $\left.\bar{n}=\left\langle n_{l}: l<\omega\right\rangle, n_{l}<n_{l+1}<\omega\right)$.

Let us carry out the induction.

CASE 1: $n=0$. Necessarily $\gamma_{n}^{j}=\lambda^{+j}$. Note that $\alpha<\gamma_{n}^{j} \Rightarrow A_{\lambda, \alpha}=\emptyset$ and the sets $\left\{\gamma_{\mathbf{n}, k}^{t}: t \in \mathcal{T}_{\lambda, 0}\right\} \cap \lambda$ for $\mathbf{n}<\mathbf{n}^{t^{*}}$ and $k<\omega$ are pairwise disjoint. Hence, the proof is easy (similar to [Sh 592, Lemma 2.14]).

CASE 2: $n+1$ and $\gamma_{n+1}^{j}>\gamma_{n}^{j}+1$. Similar to Case 1 .

CASE 3: $n+1$ and $\gamma_{n+1}^{j}=\gamma_{n}^{j}+1$. Let $\mathbf{n}$ be such that $\gamma_{\mathbf{n}, k}^{j}=\gamma_{n}^{j}$ for $k<\omega$. If $\mathbf{n} \in \operatorname{dom}\left(h_{0}\right)$ there is nothing to do, so assume $\mathbf{n} \in \operatorname{dom}\left(h_{1}\right)$. We would like to repeat the proof of [Sh 592, Lemma 2.16(1)], but this proof needs, in our notation, not just $\mathbb{P}_{\lambda^{+j}, A_{\gamma_{n}^{j}}} \lessdot \mathbb{P}_{\lambda^{+j}, \gamma_{n}^{j}}^{\prime}$ (which we have proved) but also

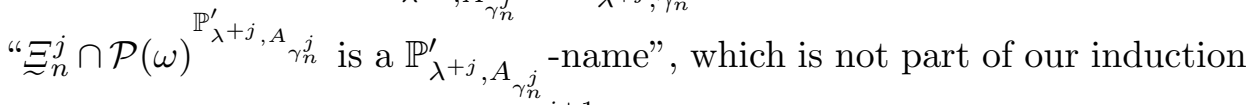
hypothesis here. But we have ${\underset{\sim}{\Xi}}_{n}^{j+1}$ in our induction hypothesis. We let $\chi$ be large enough and choose elementary submodels $M_{0}, M_{1}$ of $\left(\mathcal{H}(\chi), \in,<_{\chi}^{*}\right)$ of cardinality $\lambda^{+j}$ such that $\lambda^{+j} \subseteq M_{l},\left\{\overline{\mathbb{Q}}^{\lambda^{j+1}}, \Xi_{n}^{j+1}, \mathcal{T}_{\lambda^{+j+1}, \gamma_{n}^{j}}^{1} \subseteq M_{l}\right.$ and $M_{0} \in M_{1}$.

Now we can find a one-to-one function $h$ from $\lambda^{+j}+\kappa$ onto $\left(\lambda^{+j+1}+\kappa\right)$ $\cap M_{1}$ such that:

(1) For $\xi<\kappa, h\left(\lambda^{+j}+\xi\right)=\lambda^{+j+1}+\xi$.

(2) For $\lambda \in \lambda^{+j}$ (and $\beta \in\left[\lambda^{+j+1}, \gamma_{n}^{j}\right)$ ) we have

$$
\gamma \in A_{\lambda^{+j}, \beta} \Leftrightarrow h(\gamma) \in A_{\lambda^{+j+1}, h(\beta)}
$$

(3) For $\lambda<\lambda^{+j}$ and $n<\omega$ we have $\gamma \in B_{\lambda^{+j}, n} \Leftrightarrow h(\gamma) \in B_{\lambda^{+j+1}, n}$.

(4) For $\gamma<\lambda^{+j}$ we have $\gamma \in A_{\lambda^{+j}, \gamma_{n}^{j}} \Leftrightarrow h(\gamma) \in M_{0}$. 
This should be clear. So we have finished the induction step in constructing the $\Xi_{n^{*}}^{j}$, so we have completed the missing point in the proof of the parallel of [Sh 592, 3.4], hence of the parallel to [Sh 592, 3.3], so our main theorem is proved.

Alternative proof. Let $\overline{\mathbb{Q}}^{*}=\left\langle\mathbb{P}_{\alpha}, \mathbb{Q}_{\beta}, A_{\beta}^{*}, \tau_{\beta}: \alpha<\lambda^{+n^{*}}+\kappa\right\rangle$ be as above except that $A_{\beta}^{*}$ is $\emptyset$ if $\beta<\lambda^{+n^{*}}$ and $\left\{\gamma<\lambda^{+n^{*}}: \beta-\lambda \notin E_{\gamma}^{\lambda^{+n^{*}}}\right\} \cup[\lambda, \beta)$ if $\beta \in$ $\left[\lambda^{+n^{*}}, \lambda^{+n^{*}}+\kappa\right) \backslash\left\{\gamma_{n}: n<n^{*}\right\}$ and $\left\{\gamma<\lambda^{+n^{*}}: \beta-\lambda \notin E_{\gamma}^{\lambda^{+n^{*}}}\right\} \cup\left[\lambda^{+n^{*}}, \beta\right)$ if $\beta=\lambda+n^{*}+\gamma_{n}$.

It suffices to prove that we do not have $\left\langle\lambda^{+n}+\gamma_{l}: l<n^{*}\right\rangle, \bar{\varepsilon},\left\langle p_{l}^{\prime}: l<\omega\right\rangle$ as above for the $\overline{\mathbb{Q}}^{*}$ defined above. We take $k=n^{*}$.

[Why? Choose $\chi^{*} \gg \chi$ and an elementary submodel $M$ of $\left(\mathcal{H}\left(\chi^{*}\right), \in,<_{\chi}^{*}\right)$ of cardinality $\lambda$ to which $\overline{\mathbb{Q}}^{*}$ belongs, $M^{\omega} \subseteq M$. Now $\overline{\mathbb{Q}}^{*}$ as interpreted in $M$ is just $\overline{\mathbb{Q}}^{\lambda}$ with changes to names, and we have enough absoluteness.]

Now, fixing $\left\langle\gamma_{l}: l<n^{*}\right\rangle$ (not the others!), by induction on $n \leq n^{*}$ we prove that we can find suitable

$$
\left\langle\eta_{\beta}: \beta<\lambda^{+n^{*}}+\gamma_{n}+1+\kappa\right\rangle, \quad\left\langle\left\langle\Xi_{\alpha}^{t}: \alpha<\lambda^{+n^{*}}+\gamma_{n}+1\right\rangle: t \in T\right\rangle,
$$

and a $\mathbb{P}_{\lambda^{+n^{*}}+\gamma_{n}}$-name $\underset{\sim}{\Xi}$ (stipulating $\gamma_{0}+1=\gamma_{0}$ and $\gamma_{n^{*}}=\kappa$ ). Which means: letting $\mathbb{P}_{\alpha}, \mathbb{Q}_{\beta}, A_{\beta}, \mu_{\beta}, \tau_{\beta}$ be as in $\overline{\mathbb{Q}}^{*}$ there are $\eta_{\beta}, \Xi_{\beta}^{t}$ such that

$$
\left\langle\mathbb{P}_{\alpha}, \mathbb{Q}_{\beta}, A_{\beta}, \mu_{\beta}, \tau_{\beta}, \eta_{\beta},\left(\Xi_{\sim}^{t}\right)_{t \in \mathcal{T}}: \alpha \leq \lambda^{+n^{*}}+\gamma_{n}, \beta<\lambda^{+n^{*}}+\gamma_{n}\right\rangle \in \mathcal{K}_{\theta}^{3},
$$

i.e., it satisfies [Sh 592, Definition 2.11] except that in clause (d) there we demand that if $\mathbf{n} \in \operatorname{dom}\left(h_{1}^{t}\right)$ and $\left\langle\eta_{\mathbf{n}, l}^{t}: l<\omega\right\rangle$ is constant, then $\alpha_{l} \in$ $\left\{\gamma_{m}: n \leq m<n^{*}\right\}$. For $n$ we use $n+1$, Löwenheim-Skolem argument and uniqueness in Definition 2.11 of [Sh 592].

In detail: for $n=0$, just let $\underset{\sim}{\Xi}$ be a $\mathbb{P}_{\lambda+n^{*}+\gamma_{0}}$-name for a finitely additive measure on $\omega$ (can be Ramsey if $\mathbf{V}=\mathrm{CH}$ ) such that conditions $2.11(\mathrm{e})+(\mathrm{f})$ in [Sh 592] are satisfied. For $n$, by the induction hypothesis we have $\left\langle\eta_{\beta}^{n}\right.$ : $\left.\beta<\lambda^{+n^{*}}+\gamma_{n}\right\rangle,\left\langle\left\langle\Xi_{\alpha}^{t}: \alpha \leq \lambda^{+n^{*}}+\gamma_{n}\right\rangle: t \in \mathcal{T}\right\rangle$, we can find $A \subseteq \lambda^{+n^{*}}+\gamma_{n}$ of cardinality $\lambda^{+n}$ such that $\bigcup_{m \geq n} A_{\gamma_{m}}^{*} \subseteq A,\left[\lambda^{+n^{*}}, \lambda^{+n^{*}}+\gamma_{n}\right) \subseteq A, \mathbb{P}_{A}^{\prime} \lessdot$ $\mathbb{P}_{\lambda^{+n^{*}}+\gamma_{n}}$ and $t \in \mathcal{T} \Rightarrow \Xi_{\lambda^{+n^{*}}+\gamma_{n}}^{t}\left\lceil\mathcal{P}(\omega)^{\mathbf{V}^{\mathbb{P}_{A}^{\prime}}}\right.$ is a $\mathbb{P}_{A}^{\prime}$-name. If $A=\lambda^{+n^{*}}$, then as in [Sh 592], we can define $\underset{\sim}{\Xi_{\lambda+\gamma_{n}+1}^{t}}$ as required. This is not necessarily true, but some $f \in \operatorname{EAUT}\left(\bar{a}\left\lceil\lambda^{+n^{*}}+\gamma_{n}\right)\right.$ maps $A$ onto $A_{\lambda^{+n^{*}}, \lambda+\gamma_{n}}$, and maps $A_{\lambda^{+n^{*}}, \beta}$ (for $\beta \neq \lambda^{+n^{*}}+\gamma_{n}$ ) onto itself, so possibly changing the $\Xi_{\lambda^{+n^{*}}+\gamma_{n}}^{t}$, $\eta_{\beta}\left(\beta<\lambda^{+n^{*}}+\gamma_{n}\right)$ we can define $\underset{\sim}{\Xi_{\alpha}}$ (for $\alpha \leq \lambda^{+n^{*}}+\gamma_{n}+1$ ), $\eta_{\beta}$ (for $\left.\beta \leq \lambda^{+n^{*}}+\lambda^{+n^{*}}\right)$. The advance from $\lambda^{+n^{*}}+\gamma_{n}+1$ to $\lambda^{+n^{*}}+\gamma_{n+1}$ is as for $n=0$ : as we are proving $\in \mathcal{K}_{\theta}^{\prime}$ (not $\in \mathcal{K}_{3}$ ) there are no problems repeating the proof from [Sh 592].

So we have finished the proof of 1.1. $\mathbf{m}_{1.14}$ 
2. Non-null set with no non-null function. Peter Komjáth referred me to the following problem, answered below.

\subsection{THEOREM. It is consistent that}

$\oplus \quad$ there is a non-null $A \subseteq \mathbb{R}$ such that for every $f: A \rightarrow \mathbb{R}$, the function $f$ is null as a subset of the plane $\mathbb{R} \times \mathbb{R}$

provided that "ZFC + there is a measurable cardinal" is consistent.

Proof. We can use ${ }^{\omega} 2$ instead of $\mathbb{R}$. Let $\kappa$ be measurable, and $D$ a normal ultrafilter on $\kappa$. Let $\lambda=\lambda^{\aleph_{0}}, \lambda \geq 2^{\kappa}$ for simplicity, and we use the same forcing as in $\S 1$. Now we interpret the Cohen forcing $\mathbb{\mathbb { Q }}_{\alpha}$ (for $\alpha<\lambda$ ) as

$$
\begin{array}{r}
\left\{\left\langle\left(n_{l}, a_{l}\right): l<l^{*}\right\rangle: l^{*}<\omega, n_{l}<\omega, a_{l} \text { is a subset of }\left({ }^{n_{l}} 2\right) \times\left({ }^{n_{l}} 2\right),\right. \\
\left.\eta \in{ }^{n_{l}} 2 \Rightarrow\left|\left\{\nu \in{ }^{n_{l}} 2:(\eta, \nu) \in a_{l}\right\}\right| / 2^{n_{l}} \geq 1-4^{-l}\right\} .
\end{array}
$$

We interpret the generic sequence $\bar{a}^{\alpha}=\left\langle\left(n_{l}^{\alpha}, a_{l}^{\alpha}\right): l<\omega\right\rangle$ as the following null subset of the plane:

$$
\begin{gathered}
\operatorname{null}\left(\bar{a}^{\alpha}\right):=\left\{(\eta, \nu): \eta \in{ }^{\omega} 2 \text { and for infinitely many } l<\omega\right. \text { we have } \\
\left(\eta\left\lceil n_{l}^{\alpha}, \nu\left\lceil n_{l}^{\alpha}\right) \notin a_{l}^{\alpha}\right\} .\right.
\end{gathered}
$$

Let $\underset{\sim}{X^{*}}=\left\{\tau_{\lambda+i}: i<\kappa\right\}$; as in $\S 1$, it is not null (in fact everywhere).

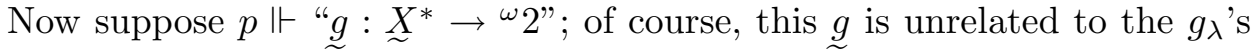
from $\S 1$. So for each $i<\kappa, g\left(\tau_{\lambda+i}\right)$ is a $\mathbb{P}_{\lambda+\kappa}$-name involving the conditions $p_{i, l} \in \mathbb{P}_{\lambda+\kappa}^{\prime}($ for $l<\omega)$. For $\tilde{\xi}<\kappa$, let $M_{\xi} \prec\left(\mathcal{H}\left(\beth_{\omega}\left(\lambda^{+\omega}\right)^{+}\right), \in,<^{*}\right)$ be such that $\overline{\mathbb{Q}},\left\langle\tau_{\lambda+i}: i<\kappa\right\rangle, p, g,\left\langle\left\langle p_{i, l}: l<\omega\right\rangle: i<\kappa\right\rangle, \lambda, \xi$ belongs to $M_{\xi}$ and $\left\|M_{\xi}\right\|=2^{\aleph_{0}}$ and ${ }^{\omega}\left(M_{\xi}\right) \subseteq M_{\xi}$, and let $w_{\xi}=M_{\xi} \cap(\lambda+\kappa)$. Then for some $A \in D$ we have:

(a) $\gamma \neq \xi \in A \Rightarrow \lambda+\xi \notin w_{\gamma}$.

(b) $\left\langle w_{\gamma}: \gamma \in A\right\rangle$ and $\left\langle M_{\gamma}: \gamma \in A\right\rangle$ form $\Delta$-systems with hearts $w^{*}, M^{*}$ respectively.

(c) $w_{\gamma} \cap[\lambda, \lambda+\gamma)$ is constant, $w_{\gamma} \cap\{\lambda+\xi: \xi \in A\}=\{\lambda+\gamma\}$, moreover $\sup \left(w_{\gamma}\right)<\lambda+\min (A \backslash(\gamma+1))$ and $w_{\gamma} \cap[\lambda, \lambda+\gamma) \subseteq w^{*}$.

(d) For $\gamma, \xi \in A$, the models $M_{\gamma}, M_{\xi}$ are isomorphic, with the isomorphism mapping $\gamma$ to $\xi$, and $\overline{\mathbb{Q}},\left\langle\tau_{\lambda+\gamma}: \gamma<\kappa\right\rangle, g$ to themselves, in fact is the identity on $M^{*}$.

So for all $\gamma \in A$ we have an isomorphic situation.

For $\gamma \in A$ let $\bar{r}^{\gamma}=\left\langle r_{\gamma, m}: m<\omega\right\rangle$ be the $<^{*}$-first maximal antichain of $\mathbb{P}_{\lambda+\kappa}$ above $p$, of forcing conditions deciding whether $\underset{\sim}{g}\left(\mathcal{\tau}_{\lambda+\gamma}\right)$ is in

$$
\mathbf{V}\left[\left\langle\tau_{\beta}: \beta \leq \lambda+\gamma+1\right\rangle\right)=\mathbf{V}^{\mathbb{P}_{\lambda+\gamma+1}},
$$

and deciding whether $g\left(\mathcal{I}_{\lambda+\gamma}\right)$ is in $\mathbf{V}\left[\left\langle\mathcal{\tau}_{\beta}: \beta \in M^{*}\right\rangle^{\wedge}\left\langle\mathcal{I}_{\lambda+\gamma}\right\rangle\right]$, and in both cases if yes, without loss of generality, it forces for some Borel function $\mathcal{B}=\mathcal{B}_{\gamma, m}$ that $r_{\gamma, m} \Vdash " g\left(\mathcal{\tau}_{\lambda+\gamma}\right)=\mathcal{B}\left(\ldots, \mathcal{I}_{\beta}, \ldots, \mathcal{I}_{\lambda+\gamma}\right)_{\beta \in w}$ ", where in the 
first case $w=w_{\gamma} \cap \gamma$ and in the second case $w=w^{*}$. As we can shrink $A$ (as long as it is in $D$ ), without loss of generality $\mathcal{B}_{\gamma, m}$ does not depend on $\gamma$, i.e. $\mathcal{B}_{\gamma, m}=\mathcal{B}_{m}$. Also, without loss of generality the answer (= decision) for each $m$ of $r_{\gamma, m}$ does not depend on $\gamma$.

Choose $\alpha<\lambda$ such that $g_{\lambda}(\alpha)=A$, where $g_{\lambda}$ is from 1.3 (and is not related to $\underset{f}{g}$ ) and $\alpha \notin \bigcup_{\gamma \in A} M_{\gamma}$. It is enough to prove the following two statements:

$(*)_{1} \quad\left\{\left(\mathcal{\tau}_{\lambda+\gamma}, g\left(\mathcal{\tau}_{\lambda+\gamma}\right)\right): \gamma \in \kappa \backslash A\right\}$ is null (subset of the plane).

$(*)_{2} \quad\left\{\left(\mathcal{\tau}_{\lambda+\gamma}, \underset{g}{g}\left(\mathcal{\tau}_{\lambda+\gamma}\right)\right): \gamma \in A\right\}$ is null.

Proof of $(*)_{1}$. Trivial as $\left\{\tau_{\lambda+\gamma}: \gamma \in \kappa \backslash A\right\}$ is null (as proved in 1.13).

Proof of $(*)_{2}$. We shall use the proof of Fact 1.14 and the choice of $\alpha$ to show that

$$
\left\{\left(\mathcal{\tau}_{\lambda+\gamma}, \underset{\sim}{g}\left(\mathcal{\tau}_{\lambda+\gamma}\right)\right): \gamma \in A\right\} \subseteq \bigcup_{l}\left({ }^{\omega_{2}} 2 \times{ }^{\omega_{2}} \backslash \backslash \lim \operatorname{tree}_{l}\left(\underline{\bar{a}}^{\alpha}\right)\right)
$$

where

$$
\operatorname{tree}_{l^{*}}\left(\overline{\bar{a}}^{\alpha}\right)=\left\{( \eta , \nu ) \in { } ^ { \omega } 2 \times { } ^ { \omega } 2 : \text { if } l \in [ l ^ { * } , \omega ) \text { then } \left(\eta\left\lceil n_{l}, \nu\left\lceil n_{l}\right) \in a_{l}^{\alpha}\right\} .\right.\right.
$$

Let ${\underset{\sim}{A}}^{m}=\left\{\xi: r_{\xi, m} \in G_{\mathbb{P}_{\lambda+\kappa}}\right\}$ for $\xi<\kappa$.

It suffices to prove, for each $m$, that

$(*)_{2, m} \quad p \Vdash$ " $\left\{\left(\mathcal{\tau}_{\lambda+\gamma}, \underset{\sim}{g}\left(\mathcal{\tau}_{\lambda+\gamma}\right)\right): \gamma \in \underset{\sim}{A}{ }^{m}\right\}$ is null".

We do it by cases (note that in each case "for some $\gamma \in A^{m}$ " is equivalent to "for every $\gamma \in{\underset{\sim}{A}}^{m}$ ").

CASE 1: $r_{\gamma, m} \Vdash " g\left(\tau_{\lambda+\gamma}\right)$ is in $\mathbf{V}\left[\left\langle\tau_{\beta}: \beta \in M^{*}\right\rangle^{\wedge}\left\langle\tau_{\lambda+\gamma}\right\rangle\right] "$. Clearly $(*)_{2, m}$ holds as the graph of a Borel function is null and we apply this to the function $\varrho \mapsto \mathcal{B}\left(\ldots, \tau_{\beta}, \ldots, \ldots ; \varrho\right)_{\beta \in M^{*}}$.

CASE 2: $r_{\gamma, m} \Vdash$ “ $\left(\mathcal{\tau}_{\lambda+\gamma}, g\left(\mathcal{\tau}_{\lambda+\gamma}\right)\right) \in\left({ }^{\omega} 2 \times{ }^{\omega} 2 \backslash \bigcup_{l<\omega} \lim \operatorname{tree}_{l}\left(\bar{a}^{\alpha}\right)\right) "$. As the set on the right side is null this is trivial.

CASE 3: Not Case 2 and $r_{\gamma, m} \Vdash " g\left(\tau_{\lambda+\gamma}\right)$ is not in $\mathbf{V}^{\mathbb{P}_{\lambda+\gamma+1}}$ "; remember $\alpha<\lambda$ was chosen such that $g_{\lambda}(\alpha)=\widetilde{A}$.

Fix $\xi=\min (A)$ and let $\xi_{1}=\min (A \backslash(\xi+1))$. Let $p^{*} \in \mathbb{P}_{\lambda+\xi_{1}}$ and $l^{*}<\omega$ be such that $r_{\xi, m} \leq p^{*}$ and $p^{*} \Vdash "\left(\mathcal{\tau}_{\lambda+\xi}, g\left(\tau_{\lambda+\gamma}\right)\right) \in \lim$ tree $l^{*}\left(\bar{a}^{\alpha}\right) "$ (note: both are $\mathbb{P}_{\lambda+\xi_{1}}$-names and $\left.r_{\xi, m} \in \mathbb{P}_{\lambda+\xi_{1}}\right)$.

As in the proof of 1.14 , we can for $\zeta<\left(2^{\aleph_{0}}\right)^{+}$by induction on $\zeta$ choose $f_{\zeta}, a_{\zeta}, p_{\zeta}$ such that

$$
f_{\zeta} \in \operatorname{EAUT}\left(\overline{\mathbb{Q}} \uparrow\left(\lambda+\xi_{1}\right)\right), \quad \alpha_{\zeta}=f_{\zeta}(\alpha), \quad \alpha_{\zeta} \notin\left\{\alpha_{\zeta_{1}}: \zeta_{1}<\zeta\right\}
$$

and $p_{\zeta}=\widehat{f}_{\zeta}\left(p^{*}\right)$ and $f_{\zeta} \uparrow\left(\bigcup_{\gamma \in A} M_{\gamma} \cap\left(\lambda+\xi_{1}\right)\right), f_{\zeta} \uparrow\left\lceil\lambda, \lambda+\xi_{1}\right)$ are the identity. So $\widehat{f_{\zeta}}$ maps $\tau_{\lambda+\xi}, \underset{\sim}{g}\left(\tau_{\lambda+\xi}\right)$ to themselves. Let $G_{\mathbb{P}_{\lambda+\xi+1}} \subseteq \mathbb{P}_{\lambda+\xi+1}$ be generic over $\mathbf{V}$ such that $\tilde{E}=\left\{\zeta: p_{\zeta} \uparrow(\lambda+\xi+1) \in G_{\mathbb{P}_{\lambda+\xi+1}}\right\}$ is unbounded in 
$\left(2^{\aleph_{0}}\right)^{+}$. So in $\mathbf{V}\left[G_{\mathbb{P}_{\lambda+\xi+1}}\right]$ we have, for $\zeta \in E$, a $\left(\mathbb{P}_{\lambda+\xi_{1}} / G_{\mathbb{P}_{\lambda+\xi+1}}\right)$-name of a real $\underset{\sim}{g}\left(\tau_{\lambda+\gamma}\right)$ which is not in $\mathbf{V}^{\mathbb{P}_{\lambda+\xi+1}}$ and

$$
p_{\zeta} \Vdash "\left(\tau_{\lambda+\xi}, \underset{\sim}{g}\left(\tau_{\lambda+\xi}\right)\right) \in \lim \operatorname{tree}_{l^{*}}\left(\bar{a}^{\alpha_{\zeta}}\right) " .
$$

Now, still as in the proof of 1.14 , we can prove by induction on $\beta \in\left[\xi+1, \xi_{1}\right]$ that in $\mathbf{V}^{\mathbb{P}_{\beta}}$, no $\left(\eta_{0}, \eta_{1}\right) \in\left({ }^{\omega} 2 \times{ }^{\omega_{2}}\right)^{V^{\mathbb{P} \lambda+\beta}} \backslash\left({ }^{\omega_{2}} \times{ }^{\omega}{ }_{2}\right)^{\mathbf{V}^{\mathbb{P}} \lambda+\xi+1}$ belongs to $\lim$ tree $_{l^{*}}\left(\bar{a}^{\alpha_{\zeta}}\right)$ for unboundedly many $\zeta \in E$. The induction is straightforward; for the successor case $\beta+1$ use $\beta \notin A=g_{\lambda}\left(\alpha_{\zeta}\right)$ (and $\widehat{f}$ for some $f \in \operatorname{EAUT}(\overline{\mathbb{Q}} \curlyvee(\lambda+i)))$. This gives us a contradiction as $p^{*}$ forces that $\left(\mathcal{\tau}_{\lambda+\xi}, g\left(\mathcal{\tau}_{\lambda+\xi}\right)\right)$ is not in $\left(\omega_{2} \times{ }^{\omega_{2}}\right)^{\mathbf{V}^{\mathbb{P}} \lambda+\xi+1}$ as the second coordinate is not in $\mathbf{V}^{\mathbb{P}} \widetilde{\lambda+\xi+1}$.

CASE 4: Neither Case 1 nor Case 2, nor Case 3. As in Case 3, but only $f_{\zeta} \uparrow\left(\left(\lambda+\xi_{1}\right) \cap M^{*}\right)$ is the identity. Hence $\widehat{f}_{\zeta} \uparrow\left(\mathbb{P}_{\lambda+\xi_{1}} \cap M^{*}\right)$ is the identity. We let

$$
B_{1}=\left\{\alpha: \alpha<\lambda+\xi_{1} \text { and } \alpha \notin M_{\xi} \cap(\lambda+\xi) \backslash M^{*}\right\} ;
$$

using $\widehat{f}$ for $f \in \operatorname{EAUT}(\overline{\mathbb{Q}})$ we can easily show that $\mathbb{P}_{B_{1}}^{\prime} \lessdot \mathbb{P}_{\lambda+\kappa}^{\prime}$, and $G_{B_{1}}=$ $G_{\mathbb{P}_{\lambda+\xi+1}} \cap \mathbb{P}_{B_{1}}^{\prime}$ (or see $\S 3$ ). Now in $\mathbf{V}\left[G_{\mathbb{P}_{\lambda+\xi+1}}\right]$ we have

$$
\underset{\sim}{E}\left[G_{B_{1}}\right]=\left\{\zeta: p_{\zeta} \uparrow(\lambda+\xi+1) \in G_{B_{1}}\right\} \text { is unbounded in }\left(2^{\aleph_{0}}\right)^{+} .
$$

We can assume that $p_{\zeta} \Vdash " g\left(\mathcal{\tau}_{\lambda+\xi}\right) \notin \mathbf{V}\left[G_{B_{1}}\right] "$ and $p_{\zeta} \in \mathcal{I}_{\bar{\varepsilon}}$ for some $\bar{\varepsilon}$ (as in $\S 1$ ), because if not then without loss of generality $p_{\zeta}$ forces $g\left(\tau_{\lambda+\xi}\right)=\tau^{\prime}$ such that $\tau^{\prime}=\mathcal{B}\left(\ldots, \text { truth value }\left(\xi_{l}^{*} \in \mathcal{\tau}_{\beta_{l}^{*}}\right), \ldots\right)_{l<\omega}$ and $\beta_{l}^{*} \in B_{1}$ and then we can find $f \in \operatorname{EAUT}(\overline{\mathbb{Q}})$ such that $\widehat{f}\left(g\left(\tau_{\lambda+\xi}\right)\right)=g\left(\tau_{\lambda+\xi}\right) \in M^{*}, \widehat{f}\left(p_{\zeta}\right) \in M_{\zeta}$ is compatible with $p_{\zeta}$, and we get an easy contradiction.

Note: in $\mathbf{V}\left[\left\langle\tau_{\beta}: \beta \in B_{1}\right\rangle\right]$ we can compute $\underset{\sim}{E}\left[G_{\mathbb{P}_{\lambda+\xi+1}}\right]$, but we do not have $g\left(\mathcal{\tau}_{\lambda+\xi}\right)$ by the previous sentence so we get an easy contradiction as in the proof of 1.14 .

3. The $\mathbb{L}_{\aleph_{1}, \aleph_{1}}$-elementary submodels and the partial FS support iteration with non-transitive memory of definable forcing. We may wonder what is really needed in $\S \S 1,2$ (and [Sh 592, $\S \S 2,3]$ ). Here we generalize one feature: iterating with partial memory but without transitivity in the memory, not restricting ourselves to Cohen and random.

3.1. Definition. $\mathcal{K}_{\kappa}^{s}$ is the family of sequences of $\overline{\mathbb{Q}}$ of the form

$$
\overline{\mathbb{Q}}=\left\langle\mathbb{P}_{\alpha}, \underset{\sim}{\mathbb{Q}_{\alpha}}, A_{\alpha}, B_{\alpha}, \bar{\mu}_{\alpha}, \bar{\varphi}_{\alpha}, \underline{\sim}_{\alpha}, \tau_{\alpha}: \alpha<\beta\right\rangle
$$

(we write $\beta=\lg (\overline{\mathbb{Q}})$; the $\varphi$ 's are absolute formulas) such that:

(a) $\left\langle\mathbb{P}_{\alpha}, \mathbb{Q}_{\alpha}: \alpha<\beta\right\rangle$ is a FS iteration of c.c.c. forcing notions (so $\mathbb{P}_{\beta}=$ $\operatorname{Lim}(\overline{\mathbb{Q}})$ denotes the limit). 
(b) $\mu_{\alpha}<\kappa$ (see clause (d) below), $\tau_{\alpha}$ is a $\mathbb{Q}_{\alpha}$-name (i.e. a $\mathbb{P}_{\alpha+1}$-name of one), $\Vdash_{\mathbb{P}_{\alpha+1}}$ " $\tau_{\alpha} \subseteq \mu_{\alpha}$ and $\tau_{\alpha}$ is the generic of $\mathbb{Q}_{\alpha}$ ".

(c) $B_{\alpha} \subseteq A_{\alpha} \subseteq \alpha$ and $\beta \in B_{\alpha} \Rightarrow B_{\beta} \subseteq B_{\alpha}$ and $\left|B_{\alpha}\right|<\kappa$.

First simple version:

(d) $\bar{\mu}=\left\langle\mu_{\alpha}^{0}, \mu_{\alpha}^{1}, \mu^{2}\right), \bar{\varphi}=\left\langle\varphi_{\alpha}^{0}, \varphi_{\alpha}^{1}\right\rangle, \varphi_{\alpha}^{0}=\varphi_{\alpha}^{0}\left(x, \underline{\sim}_{\alpha}\right)$, and $\varphi_{\alpha}^{1}=\varphi_{\alpha}^{1}\left(x, y, \underline{\sim}_{\alpha}\right)$; let $\mu_{\alpha}=\mu_{\alpha}^{2}$.

(e) The set of elements of $\mathbb{Q}_{\alpha}$ is a non-empty subset of $\mathbb{Q}_{\alpha}^{\text {pos }}=\{\eta: \eta \in$ $\left.\omega\left(\mu_{\alpha}^{0}\right)\right\}$ in the universe $\mathbf{V}\left[\left\langle\tau_{\gamma}: \tilde{:} \gamma \in A_{\alpha}\right\rangle\right]$.

(f) $\underline{\sim}_{\alpha}$ is a $\mathbb{P}_{\alpha}$-name of a subset of $\mu_{\alpha}^{1}$, moreover we have sequences $\overline{\mathcal{B}}_{\alpha}=\left\langle\mathcal{B}_{\alpha, \zeta}: \zeta<\mu_{\alpha}^{1}\right\rangle, \bar{\xi}_{\alpha}=\left\langle\xi_{\alpha, \zeta, n}: \zeta<\mu_{\alpha}^{1}, n<\omega\right\rangle$ and $\bar{\beta}_{\alpha, \zeta}=\left\langle\beta_{\alpha, \zeta, n}:\right.$ $\left.\zeta<\mu_{\alpha}^{1}, n<\omega\right\rangle$, all three from $\mathbf{V}$, such that:

$(\alpha) \mathcal{B}_{\alpha, \zeta}=\mathcal{B}_{\alpha, \zeta}\left(\ldots, x_{n}, \ldots\right)_{n<\omega}$ is a Borel function from ${ }^{\omega}\{$ true, false $\}$ to $\{$ true, false ,

(B) $\xi_{\alpha, \zeta, n}<\mu_{\beta_{\alpha, \zeta, n}}, \beta_{\alpha, \zeta, n} \in B_{\alpha}$,

$(\gamma) \underset{\sim}{Y_{\alpha}} \in \mathbf{V}\left[\left\langle\tau_{\gamma}: \gamma \in B_{\alpha}\right\rangle\right)$ is the set

$\left\{\zeta<\mu_{\alpha}^{1}: \mathcal{B}_{\alpha, \zeta}\left(\ldots, \text { truth value }\left(\xi_{\alpha, \zeta, n} \in \mathcal{I}_{\beta_{\alpha, \zeta, n}}\right), \ldots\right)_{n<\omega}=\operatorname{truth}\right\}$.

(g) $p \in{\underset{\sim}{\mathbb{Q}} \alpha}_{\alpha}$ iff $\left\{\eta \in{\underset{\sim}{\mathbb{Q}}}_{\alpha}^{\text {pos }}: \mathbf{V}\left[\left\langle\tau_{\gamma}: \gamma \in B_{\alpha}\right\rangle\right] \models \varphi_{\alpha}^{0}(p, \underset{\sim}{Y})\right\}$ and

$$
\mathbb{Q}_{\alpha}=p \leq q \quad \text { iff } \quad \mathbf{V}\left[\left\langle\tau_{\gamma}: \gamma \in B_{\alpha}\right\rangle\right] \models \varphi_{\alpha}^{1}[p, q, \underset{\sim}{Y}] .
$$

Second, non-simple version:

(d) $\bar{\mu}_{\alpha}=\left\langle\mu_{\alpha}^{l}: l<7\right\rangle$; we let $\mu_{\alpha}=\mu_{\alpha}^{4}, \bar{\varphi}_{\alpha}=\left\langle\varphi_{\alpha}^{l}: l<6\right\rangle, \varphi_{\alpha}=\varphi_{\alpha}^{0}$, $\varphi_{\alpha}^{l}$ is a 3-place relation on ${ }^{\omega>}\left(\mu_{\alpha}^{l}\right)$ if $l=0,3$, a 4-place relation on ${ }^{\omega>}\left(\mu_{\alpha}^{l}\right)$ if $l=1,2, \varphi_{\alpha}^{4}$ is binary, $\varphi_{\alpha}^{5}$ is a 5-place relation, and for $i<6, \varphi_{\alpha}^{i}$ is a subset $\left({ }^{4}\right)$ of $\left\{\bar{\eta}: \bar{\eta}=\left(\eta_{0}, \ldots, \eta_{j-1}\right)\right.$, for some $n$ each $\eta_{l}$ is a sequence of ordinals of length $n$, and $(i, j) \in\{(0,3),(1,4),(2,4),(3,3),(4,2),(5,5)\}\}$ (can read more natural restrictions), $\varphi_{\alpha}^{i}$ is closed under initial segments (i.e. letting $i=0, \bar{\eta}=\left(\eta_{0}, \eta_{1}, \eta_{2}\right) \in \varphi_{\alpha}^{0} \Rightarrow \bar{\eta}\left\lceil n=\left(\eta_{0}\left\lceil n, \eta_{1}\left\lceil n, \eta_{2}\lceil n) \in \varphi_{\alpha}^{0}\right.\right.\right.\right.$, of course $\bar{\eta}\lceil n$ is an abuse of notation),

$\lim \left(\varphi_{\alpha}\right)=\{(\eta, \nu, \varrho): \eta, \nu, \varrho$ are sequences of length $\omega$

$$
\text { such that } n<\omega \Rightarrow\left(\eta \left\lceiln, \nu\left\lceil n, \varrho\lceil n) \in \varphi_{\alpha}\right\}\right.\right.
$$

(depend on the universe) and similarly for other $\varphi_{\alpha}^{i}$.

(e) The set of elements of $\mathbb{Q}_{\alpha}$ is a (non-empty) subset of

$$
\mathbb{Q}_{\alpha}^{\text {pos }}=\left\{\eta: \eta \in{ }^{\omega}\left(\mu_{\alpha}^{0}\right) \cap \mathbf{V}\left[\mathcal{I}_{\gamma}: \gamma \in A_{\alpha}\right]\right\} .
$$

$\left({ }^{4}\right)$ Note: $\lim \left(\varphi_{\alpha}^{0}\right)$ describes $\mathbb{Q}_{\alpha}, \lim \left(\varphi_{\alpha}^{1}\right), \lim \left(\varphi_{\alpha}^{2}\right)$ describe $\leq_{\mathbb{Q}_{\alpha}},+_{\mathbb{Q}_{\alpha}}, \lim \left(\varphi_{\alpha}^{3}\right)$ describes the maximal antichain of $\mathbb{Q}_{\alpha}, \lim \left(\varphi_{\alpha}^{4}\right)$ describes " $\xi \in \tau_{\alpha}$ " and $\lim \left(\varphi_{\alpha}^{5}\right) \operatorname{describes}$ $G_{\alpha}(2)$. 
(f) $\underline{\varrho}_{\alpha}=\left\langle\varrho_{\alpha, \zeta}^{l}: \zeta<\zeta_{\alpha}^{l}, l<6\right\rangle, \varrho_{\alpha, \zeta}^{l}=\left\langle\varrho_{\alpha, \zeta, m}^{l}: m<\omega\right\rangle$, and $\varrho_{\alpha, \zeta, m}^{l}$ is a $\mathbb{P}_{\alpha}$-name of a natural number (or of a member of $\{0,1\}$ ), it has the form $\mathcal{B}_{\alpha, \zeta, m}^{l}\left(\ldots\right.$, truth value $\left.\left.\left(\xi_{\alpha, \zeta, m, k}^{l} \in \mathcal{\tau}_{\beta_{\alpha, \zeta, m, k}^{l}}\right), \ldots\right)\right)_{k<\omega}, \mathcal{B}_{\alpha, \zeta, m}^{l}$ is a Borel function, $\beta_{\alpha, \zeta, m, k}^{l} \in B_{\alpha}$ and $\xi_{\alpha, \zeta, m, k}^{l}<\mu_{\alpha}$.

(g) In $\mathbf{V}\left[\left\langle\tau_{\beta}: \beta \in A_{\alpha}\right\rangle\right], \mathbb{Q}_{\alpha}$ is the set of elements of $\left\{\eta \in \mathbb{Q}_{\alpha}^{\text {pos }}\right.$ : there are $\nu \in{ }^{\omega}(\mathrm{Ord})$ and $\zeta<\zeta_{\alpha}^{0}$ such that $\left(\eta, \nu, \varrho_{\alpha, \zeta}^{0}\right) \in \lim \left(\varphi_{\alpha}^{0}\right)$ and $\nu(0)=\zeta$; so $\eta, \nu, \varrho_{\alpha, \zeta}^{0}\left[G_{\mathbb{P}_{\alpha}}\right]$ belongs to $\left.\mathbf{V}\left[\left\langle\tau_{\beta}: \beta \in A_{\alpha}\right\rangle\right]\right\}$; we may write $\zeta=\zeta_{\alpha}^{0}(p)$. Similarly $\leq_{\mathbb{Q}_{\alpha}}$ is defined by $\varphi_{\alpha}^{1}$.

(h) In $\tilde{\mathbf{V}}\left[\left\langle\tau_{\beta}: \beta \in A_{\alpha}\right\rangle\right]$ we have $\left\{(p, q): p \leq_{\mathbb{Q}_{\alpha}} q\right\}=\left\{(p, q): p, q \in \mathbb{Q}_{\alpha}\right.$

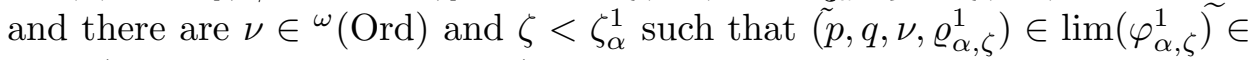
$\left.\lim \left(\varphi_{\alpha}^{1}\right), \nu(0)=\zeta\right\}$. We write $\zeta_{\alpha}^{1}(p, q)$ for the minimal $\zeta$.

(i) In $\mathbf{V}\left[\left\langle\tau_{\beta}: \beta \in A_{\alpha}\right\rangle\right]$ we have $\left\{(p, q): p, q \in \mathbb{Q}_{\alpha}\right.$ are compatible in $\left.\mathbb{Q}_{\alpha}\right\}=\left\{(p, q): p, q \in \mathbb{Q}_{\alpha}\right.$ and there are $\nu \in{ }^{\omega}($ Ord $)$ and $\zeta<\zeta_{\alpha}^{2}$ such that $\left.\widetilde{(p}, q, \nu, \varrho_{\alpha, \zeta}^{2}\right) \in \lim \left(\varphi_{\alpha}^{2} \widetilde{)}\right.$ and $\left.\nu(0)=\zeta\right\}$ (we write $\zeta_{\alpha}^{2}(p, q)$ for the minimal such $\zeta)$.

(k) In $\mathbf{V}\left[\left\langle\tau_{\beta}: \beta \in A_{\alpha}\right\rangle\right]$ we have $\left\{\left\langle p_{n}: n<\omega\right\rangle: p_{n} \in \mathbb{Q}_{\alpha}\right.$ for $n<\omega$, $\left\{p_{n}: n<\omega\right\}$ is predense in $\left.\mathbb{Q}_{\alpha}\right\}=\left\{\left\langle p_{n}: n<\omega\right\rangle\right.$ : for some $\nu^{\omega}{ }^{\omega}($ Ord $)$ and $\zeta<\zeta_{\alpha}^{3}$ we have $\left(p, \nu, \varrho_{\alpha, \zeta}^{3}\right) \in \widetilde{\lim }\left(\varphi_{\alpha}^{3}\right)$ [where $\left.\left.p=\left\langle p_{n-[\sqrt{n}]^{2}}([\sqrt{n}]): n<\omega\right\rangle\right]\right\}$, and we write $\zeta_{\alpha}^{2}(\bar{p})$ for the minimal such $\zeta$.

(l) In $\mathbf{V}\left[\left\langle\tau_{\beta}: \beta \in A_{\alpha}\right\rangle\right]$ we have $\left\{(p, \xi): p \in \mathbb{Q}_{\alpha}, \xi<\mu_{\alpha}^{4}\right.$ and $p \Vdash_{\mathbb{Q}_{\alpha}}$ " $\xi \in$ $\left.\tau_{\alpha} "\right\}=\left\{(p, \xi)\right.$ : for some $\nu \in{ }^{\omega}\left(\mu_{\alpha}^{4}\right)$ and $\zeta<\zeta_{\alpha}^{4} \widetilde{\text { we have }}\left\langle\langle\xi\rangle^{\wedge} p,\langle\zeta\rangle \tilde{\epsilon} \varphi_{\alpha}^{4}\right\}$.

(m) If $G_{<\alpha} \subseteq \mathbb{P}_{\alpha}$ is generic over $\mathbf{V}, G_{\alpha} \subseteq \mathbb{Q}_{\alpha}\left[G_{<\alpha}\right]$ is generic over $\mathbf{V}\left[G_{<\alpha}\right]$ and $\tau_{\alpha}=\tau_{\alpha}\left[G_{\alpha}\right]$, then in $\mathbf{V}\left[\left\langle\tau_{\beta}\left[G_{\alpha}\right]: \beta \in A_{\alpha}\right\rangle\right]$ we have

$$
\begin{aligned}
G_{\alpha}=\left\{\eta \in{ }^{\omega}(\text { Ord }):\right. & \text { there are } \nu \in{ }^{\omega}(\text { Ord }), \zeta<\zeta_{\alpha}^{5}, \\
& \nu^{1} \in{ }^{\omega}\left(\mu_{\alpha}\right), \text { and } \varrho \in{ }^{\omega} 2 \text { such that } \\
& \varrho(n)=0 \Leftrightarrow \nu^{1}(n) \in \tau_{\alpha} \text { and } \\
& \left.\left(\eta, \nu, \nu^{1}, \varrho, \varrho_{\alpha, \zeta}\right) \in \lim \left(\varphi_{\alpha}^{5}\right)\right\} .
\end{aligned}
$$

We can simplify Definition 3.1 by

3.2. Definition. (1) We say $\mathcal{B}$ is a Borel function to $\mathbf{V}$ if it is a function from ${ }^{\omega}\{0,1\}$ to $\mathbf{V}$ (we identify sometimes 1 with truth, 0 with false) with countable range such that each $\left\{\eta \in{ }^{\omega} 2: \mathcal{B}(\eta)=x\right\}$ is a Borel set.

(2) We say $\mathcal{B}$ is a Borel function to ${ }^{\omega} \mathbf{V}$ if $\mathcal{B}(\eta)=\left\langle\mathcal{B}_{n}(\eta): n\langle\omega\rangle\right.$ with each $\mathcal{B}_{n}$ being a Borel function to $\mathbf{V}$.

(3) $\overline{\mathbb{Q}}$ is simple if in Definition 3.1(e),

$$
\lim \left(\varphi_{\alpha}^{0}\right) \subseteq\left\{(\eta, \nu, \varrho): \varrho \in{ }^{\omega} \omega\right\} .
$$

3.3. Definition. Let $\overline{\mathbb{Q}} \in \mathcal{K}_{\kappa}^{s}$ and $\alpha=\lg (\overline{\mathbb{Q}})$. 
(1) Let

$\mathbb{P}_{\beta}^{\prime}=\left\{p \in \mathbb{P}_{\beta}\right.$ : for every $\gamma \in \operatorname{dom}(p), p(\gamma)$ is computed by some Borel function $\mathcal{B}$ (so $\mathcal{B} \in \mathbf{V}$, and is an object, not a name), $\mathcal{B}$ is a function to ${ }^{\omega} \mathbf{V}$ from some $\left\langle\right.$ truth value $\left.\left(\xi_{l} \in \tau_{\alpha_{l}}\right): l<\omega\right\rangle$ where $\xi_{l}<\mu_{\alpha_{l}}$ and $\alpha_{l}<\gamma$ and we say in this case that the truth value of $\left(\xi_{l} \in \tau_{\alpha_{l}}\right)$ appears in $p(\gamma)$ for $l<\omega$ and $p\left\lceil\gamma\right.$ forcing a value to $\left.\zeta_{\gamma}^{0}(p(\gamma))\right\}$.

(2) For $p \in \mathbb{P}_{\beta}^{\prime}$ and $\alpha \in \operatorname{dom}(p)$ let $\operatorname{supp}(p(\alpha))=\left\{\gamma\right.$ : for some $\xi$ the truth value of $\left(\xi \in \mathcal{I}_{\gamma}\right)$ appears in $\left.p(\alpha)\right\}$ and let $\operatorname{supp}(p)=\operatorname{dom}(p) \cup \bigcup_{\alpha \in \operatorname{dom}(p)} \operatorname{supp}(p(\alpha))$.

(3) For $A \subseteq \alpha$ let

$$
\mathbb{P}_{A}^{\prime}=\left\{p \in \mathbb{P}_{\alpha}^{\prime}: \operatorname{dom}(p) \subseteq A \text { and } \gamma \in \operatorname{dom}(p) \Rightarrow \operatorname{supp}(p(\gamma)) \subseteq A\right\},
$$

i.e., $\mathbb{P}_{A}^{\prime}=\left\{p \in \mathbb{P}_{\alpha}^{\prime}: \operatorname{supp}(p) \subseteq A\right\}$ with the order inherited from $\mathbb{P}_{\alpha}^{\prime}$ which is inherited from $\mathbb{P}_{A}$ (recall: only for some $A$ 's, $\mathbb{P}_{A}^{\prime} \lessdot \mathbb{P}_{\alpha}^{\prime}$ ).

(4) $A$ is called $\overline{\mathbb{Q}}$-closed if $A \subseteq \lg (\overline{\mathbb{Q}})$ and $\alpha \in A \Rightarrow B_{\alpha} \subseteq A$. We call $A$ strongly $\overline{\mathbb{Q}}$-closed if $A \subseteq \lg (\overline{\mathbb{Q}})$ and $\alpha \in A \Rightarrow A_{\alpha} \subseteq A$. We let $\operatorname{cl}_{\overline{\mathbb{Q}}}(A)$ be the $\overline{\mathbb{Q}}$-closure of $A$ and $\operatorname{scl}_{\overline{\mathbb{Q}}}(A)$ be the strong $\overline{\mathbb{Q}}$-closure of $A$.

(5) Let

Simple version:

$\operatorname{PAUT}(\overline{\mathbb{Q}})=\{f:$

(i) $f$ is a one-to-one function,

(ii) the domain and range of $f$ are $\overline{\mathbb{Q}}$-closed (in particular are $\subseteq \lg (\overline{\mathbb{Q}})$ ),

(iii) $\left(\forall \alpha_{1}, \alpha_{2} \in \operatorname{dom}(f)\right)\left(\alpha_{1} \in A_{\alpha_{2}} \Leftrightarrow f\left(\alpha_{1}\right) \in A_{f\left(\alpha_{2}\right)}\right)$

(iv) $\left(\forall \alpha_{1}, \alpha_{2} \in \operatorname{dom}(f)\right)\left(\alpha_{1} \in B_{\alpha_{2}} \Leftrightarrow f\left(\alpha_{1}\right) \in B_{f\left(\alpha_{2}\right)}\right)$

(v) if $f\left(\alpha_{1}\right)=\alpha_{2}$ then $f$ maps ${\underset{\sim}{\alpha_{1}}}_{\alpha_{1}}$ to ${\underset{\sim}{\alpha_{2}}}_{\mathcal{B}_{\alpha_{2}}}$,

i.e. $\bar{\mu}_{\alpha_{1}}=\bar{\mu}_{\alpha_{2}}, \bar{\varphi}_{\alpha_{1}}=\bar{\varphi}_{\alpha_{2}}, \mathcal{B}_{\alpha_{1}, \zeta}=\mathcal{B}_{\alpha_{2}, \zeta}$,

$\left.\xi_{\alpha_{1}, \zeta, n}=\xi_{\alpha_{2}, \zeta, n}, \beta_{\alpha_{1}, \zeta, n}=\beta_{\alpha_{2}, \zeta, n}\right\}$.

Non-simple version:

$\operatorname{PAUT}(\overline{\mathbb{Q}})=\{f:$

(i) $f$ is a one-to-one function,

(ii) the domain and range of $f$ are $\overline{\mathbb{Q}}$-closed, (in particular are $\subseteq \lg (\overline{\mathbb{Q}})$ ),

(iii) $\left(\forall \alpha_{1}, \alpha_{2} \in \operatorname{dom}(f)\right)\left(\alpha_{1} \in A_{\alpha_{2}} \Leftrightarrow f\left(\alpha_{1}\right) \in A_{f\left(\alpha_{2}\right)}\right)$,

(iv) $\left(\forall \alpha_{1}, \alpha_{2} \in \operatorname{dom}(f)\right)\left(\alpha_{1} \in B_{\alpha_{2}} \Leftrightarrow f\left(\alpha_{1}\right) \in B_{f\left(\alpha_{2}\right)}\right)$,

(v) if $f\left(\alpha_{1}\right)=\alpha_{2}$ then $\mu_{\alpha_{1}}^{l}=\mu_{\alpha_{2}}^{l}, \zeta_{\alpha_{1}}^{l}=\zeta_{\alpha_{2}}^{l}$, $\varphi_{\alpha_{1}}^{l}=\varphi_{\alpha_{2}}^{l}$ and $f$ maps $\varrho_{\alpha_{1}, \zeta}^{l}$ to $\varrho_{\alpha_{2}, \zeta}^{l}$, i.e. 


$$
\begin{aligned}
& \mathcal{B}_{\alpha_{1}, \zeta, m}^{l}=\mathcal{B}_{\alpha_{2}, \zeta, m}^{l} \text { for } l<6, \zeta<\zeta_{\alpha_{1}}^{l}=\zeta_{\alpha_{2}}^{l}, m<\omega, \\
& \text { and } \xi_{\alpha_{1}, \zeta, m, k}^{l}=\xi_{\alpha_{2}, \zeta, m, k}^{l} \text { for } l<6, \zeta<\zeta_{\alpha_{1}}^{l}=\zeta_{\alpha_{2}}^{l}, \\
& m<\omega, k<\omega, \text { but, of course, } \\
& \text { " } \xi \in \mathcal{I}_{\beta} \text { " is replaced by " } \xi \in \mathcal{I}_{f(\beta)} \text { ", } \\
& \text { that is, } \left.\beta_{\alpha_{2}, \zeta, m, k}^{l}=f\left(\beta_{\alpha_{1}, \zeta, m, k}^{l}\right)\right\} .
\end{aligned}
$$

(6) For $f \in \operatorname{PAUT}(\overline{\mathbb{Q}}), \widehat{f}$ is the natural map which $f$ induces from $\mathbb{P}_{\operatorname{dom}(f)}^{\prime}$ onto $\mathbb{P}_{\text {rang }(f)}^{\prime}$; similarly for part $(7)$.

(7) For $\overline{\mathbb{Q}}^{1}, \overline{\mathbb{Q}}^{2} \in \mathcal{K}_{\kappa}^{s}$ let PAUT $\left(\overline{\mathbb{Q}}^{1}, \overline{\mathbb{Q}}^{2}\right)$ be defined similarly:

\section{$\operatorname{PAUT}\left(\overline{\mathbb{Q}}^{1}, \overline{\mathbb{Q}}^{2}\right)=\{f$ :}

(i) $f$ is a one-to-one function,

(ii) the domain of $f$ is $\overline{\mathbb{Q}}^{1}$-closed

(in particular is $\subseteq \lg \left(\overline{\mathbb{Q}}^{1}\right)$ )

and the range of $f$ is $\overline{\mathbb{Q}}^{2}$-closed

(in particular is $\subseteq \lg \left(\overline{\mathbb{Q}}^{2}\right)$ ),

(iii) $\left(\forall \alpha_{1}, \alpha_{2} \in \operatorname{dom}(f)\right)\left(\alpha_{1} \in A_{\alpha_{2}}^{1} \Leftrightarrow f\left(\alpha_{1}\right) \in A_{f\left(\alpha_{2}\right)}^{2}\right)$,

(iv) $\left(\forall \alpha_{1}, \alpha_{2} \in \operatorname{dom}(f)\right)\left(\alpha_{1} \in B_{\alpha_{2}}^{1} \Leftrightarrow f\left(\alpha_{1}\right) \in B_{f\left(\alpha_{2}\right)}^{2}\right)$,

(v) if $f\left(\alpha_{1}\right)=\alpha_{2}$ then

simple version:

${ }^{1} \bar{\mu}_{\alpha_{1}}={ }^{2} \bar{\mu}_{\alpha_{1}}, \bar{\varphi}_{\alpha_{1}}={ }^{2} \bar{\varphi}_{\alpha_{2}}$ and $f$ maps $\underset{\sim \alpha_{1}}{Y}$ to $\underset{\sim}{Y} \alpha_{2}$,

i.e., $\mathcal{B}_{\alpha_{1}, \zeta}^{1}=\mathcal{B}_{\alpha_{2}, \zeta}^{2}, \quad \xi_{\alpha_{1}, \zeta, m}^{1}=\xi_{\alpha_{2}, \zeta, m}^{2}$,

$\beta_{\alpha_{1}, \zeta, m}^{1}=\beta_{\alpha_{2}, \zeta, m}^{2}$,

non-simple version: (for $l<6$ )

${ }^{1} \mu_{\alpha_{1}}^{l}={ }^{2} \mu_{\alpha_{2}}^{l},{ }^{1} \zeta_{\alpha_{1}}^{l}={ }^{2} \zeta_{\alpha_{2}}^{l},{ }^{1} \varphi_{\alpha_{1}}^{l}={ }^{2} \varphi_{\alpha_{2}}^{l}$ and

$f$ maps ${ }^{1} \varrho_{\alpha_{1}, \zeta}^{l}$ to ${ }^{2} \varrho_{\alpha_{2}, \zeta}^{l}$, i.e., $\mathcal{B}_{\alpha_{2}, \zeta, m}^{l}=\mathcal{B}_{\alpha_{1}, \zeta, m}^{l}$,

$\xi_{\alpha_{2}, \zeta, m, k}^{l}=\xi_{\alpha_{1}, \zeta, m, k}^{l}$ and $\beta_{\alpha_{2}, \zeta, m, k}^{l}=f\left(\beta_{\alpha_{1}, \zeta, m, k}^{l}\right)$

for $\left.\left.\zeta<\zeta_{\alpha_{1}}^{l}=\zeta_{\alpha_{2}}^{l}, m<\omega, k<\omega\right)\right\}$.

3.4. Claim. Let $\overline{\mathbb{Q}} \in \mathcal{K}_{\kappa}^{s}$ be of length $\alpha^{*}$.

(1) $\mathbb{P}_{\alpha^{*}}^{\prime}$ is a dense subset of $\mathbb{P}_{\alpha^{*}}$.

(2) In $\mathbf{V}^{\mathbb{P}_{\alpha}}$, from $\tau_{\alpha}\left[G_{\mathbb{Q}_{\alpha}}\right]$ we can reconstruct $G_{\mathbb{Q}_{\alpha}}$ and vice versa. From $\left\langle\tau_{\gamma}: \gamma<\alpha\right\rangle\left[G_{\mathbb{P}_{\alpha}}\right]$ we can reconstruct $G_{\mathbb{P}_{\alpha}}$ and vice versa.

(3) $\mathbf{V}^{\mathbb{P}_{\alpha}}=\mathbf{V}\left[\left\langle\tau_{\beta}: \beta<\alpha\right\rangle\right]$.

(4) If $\mu$ is any cardinal, and $\underset{\sim}{X}$ is a $\mathbb{P}_{\alpha^{*}}$-name of a subset of $\mu$, then there is a set $A \subseteq \alpha^{*}$ such that $|A| \leq \mu$ and $\Vdash_{\mathbb{P}_{\alpha^{*}}}$ "X $\underset{\sim}{X} \in \mathbf{V}\left[\left\langle\tau_{\gamma}: \gamma \in A\right\rangle\right]$ ". Moreover, for each $\zeta<\mu$ there is in $\mathbf{V}$ a Borel function $\mathcal{B}_{\zeta}\left(x_{0}, \ldots, x_{n}, \ldots\right)_{n<\omega}$ with domain and range the set \{true, false\} and $\gamma_{l} \in A, \xi_{\zeta, l}<\mu_{\gamma_{\zeta}}$ for $l<\omega$ (i.e. $\left.\left\langle\mathcal{B}_{\zeta}, \gamma_{\zeta, l}, \xi_{\zeta, l}: \zeta<\mu, l<\omega\right\rangle \in \mathbf{V}\right)$ such that

$\Vdash_{\mathbb{P}_{\alpha^{*}}} " \zeta \in \underset{\sim}{X}$ iff true $=\mathcal{B}_{\zeta}\left(\ldots\right.$, truth value $\left.\left(\xi_{\zeta, l} \in \mathcal{\tau}_{\gamma_{\zeta, l}}\left[G_{\mathbb{Q}_{\gamma_{\zeta, l}}}\right]\right), \ldots\right)$ ". 
Proof. (1) Easy.

(2), (3) By induction on $\alpha$.

(4) Let $\chi^{*}$ be such that $\{\overline{\mathbb{Q}}, \lambda\} \in \mathcal{H}\left(\chi^{*}\right)$, and let $\zeta<\mu$; let $M$ be an elementary submodel of $\left(\mathcal{H}\left(\chi^{*}\right), \in,<_{\chi^{*}}^{*}\right)$ to which $\{\overline{\mathbb{Q}}, \lambda, \kappa, \mu, \underset{\sim}{X}, \zeta\}$ belongs and $\mu \subseteq M$, so $\Vdash_{\mathbb{P}_{\alpha^{*}}}$ " $M\left[G_{\mathbb{P}_{\alpha^{*}}}\right] \cap \mathcal{H}\left(\chi^{*}\right)=M$ ". Hence by 3.4(3) (i.e. as $\left.\mathbf{V}^{\mathbb{P}_{\alpha}}=\mathbf{V}\left[\left\langle\tau_{\beta}: \beta<\alpha\right\rangle\right]\right)$ we have $M\left[G_{\mathbb{P}_{\alpha^{*}}}\right]=M\left[\left\langle\tau_{i}: i \in \alpha \cap M\right\rangle\right]$ and the conclusion should be clear.

3.5. Claim. For $A \subseteq \alpha^{*}$, every real in $\mathbf{V}\left[\left\langle\tau_{\gamma}: \gamma \in A\right\rangle\right]$ or even a subset $X$ of $\mu$ (for some $\mu$ ) has the form mentioned in 3.4(4) with $\gamma_{\zeta, l} \in A$.

Proof. This does not follow by 3.4(4) as e.g. maybe $\neg \mathbb{P}_{A}^{\prime} \lessdot \mathbb{P}_{\alpha}$. Let $\mathcal{L}_{\lambda^{+}, \omega}$ denote the propositional logic allowing conjunctions and disjunctions of size $\leq \lambda$. We know that if $X \subseteq \mu$ and $X \in \mathbf{V}\left[\left\langle\tau_{\gamma}: \gamma \in A\right\rangle\right]$, where $\tau_{\gamma} \subseteq \mu_{\gamma}$ (and $\left\langle\mu_{\gamma}: \gamma \in A\right\rangle \in \mathbf{V}$ ), then we can find in $\mathbf{V}$ a sequence $\left\langle\mathcal{B}_{\zeta}: \zeta<\mu\right\rangle$, $\left\langle\left(\xi_{\zeta, i}, \gamma_{\zeta, i}\right): \zeta<\mu, i<\mu_{\zeta}\right\rangle, \xi_{\gamma, i}<\mu_{\gamma_{\zeta, i}}, \mathcal{B}_{\zeta}=\mathcal{B}_{\zeta}\left(\ldots, x_{i}, \ldots\right)_{i<\mu_{\zeta}} \in \mathcal{L}_{\lambda^{+}, \omega}$ for some $\lambda$ such that

$$
X=\left\{\zeta<\mu: \mathcal{B}_{\zeta}\left(\ldots, \text { truth value }\left(\xi_{\zeta, i} \in \tau_{\gamma_{\zeta, i}}\right), \ldots\right)_{i<\mu_{i}}=\operatorname{truth}\right\} .
$$

So if $p \in \mathbb{P}_{\alpha}$ and $p \Vdash$ " $\tau \in \mu$ ", we can find $q, p \leq q \in \mathbb{P}_{\alpha}$ and

$$
\left\langle\left(\mathcal{B}_{\zeta}, \mu_{\zeta}, \xi_{\zeta, i}, \gamma_{\zeta, i}\right): \zeta<\mu, i<\mu_{\zeta}\right\rangle \in \mathbf{V}
$$

as above with $\gamma_{\zeta, i} \in A$ such that

$$
q \Vdash_{\mathbb{P}_{\alpha}} " \tau=\left\{\zeta<\mu: \mathcal{B}_{\zeta}\left(\ldots, \text { truth value }\left(\xi_{\zeta, i} \in \mathcal{I}_{\gamma, \zeta, i}\right), \ldots\right)_{i<\mu_{\zeta}}\right\} .
$$

Now as $\mathbb{P}_{\alpha}$ satisfies the c.c.c., for each $\zeta$ separately we can replace the conjunction and disjunction inside $\mathcal{B}_{\zeta}$ by countable ones, so we are done. $\mathbf{- 3}_{3.5}$

3.6. Claim. (1) If $\overline{\mathbb{Q}}^{1}, \overline{\mathbb{Q}}^{2} \in \mathcal{K}_{\kappa}^{s}$ and $f \in \operatorname{PAUT}\left(\overline{\mathbb{Q}}^{1}, \overline{\mathbb{Q}}^{2}\right)$ and $\operatorname{dom}(f)=$ $\lg \left(\overline{\mathbb{Q}}^{1}\right), \operatorname{rang}(f)=\lg \left(\overline{\mathbb{Q}}^{2}\right)$, then $\hat{f}$ is an isomorphism from $\left(\mathbb{P}_{\lg \left(\overline{\mathbb{Q}}^{1}\right)}\right)^{\left(\overline{\mathbb{Q}}^{1}\right)}$ onto

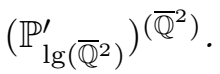

(2) If $\overline{\mathbb{Q}} \in \mathcal{K}_{\kappa}^{s}$ and $A \subseteq \lg (\overline{\mathbb{Q}})$ is strongly $\overline{\mathbb{Q}}$-closed, then $\mathbb{P}_{A}^{\prime} \lessdot \mathbb{P}_{\lg (\overline{\mathbb{Q}})}^{\prime}$, in fact if $q \in \mathbb{P}_{\lg (\overline{\mathbb{Q}})}^{\prime}$ and $q \uparrow A \leq p \in \mathbb{P}_{A}^{\prime}$, then $p \cup\left(q \uparrow(\lg (\overline{\mathbb{Q}} \backslash A)) \in \mathbb{P}_{\lg (\overline{\mathbb{Q}})}^{\prime}\right.$ is a lub of $p, q$ and there are unique $\left(\overline{\mathbb{Q}}^{\prime}, f\right)$ such that $\overline{\mathbb{Q}}^{\prime} \in \mathcal{K}_{\kappa}^{s}, f \in \operatorname{PAUT}\left(\overline{\mathbb{Q}^{\prime}}, \overline{\mathbb{Q}}\right)$, $f$ is order preserving, $\operatorname{dom}(f)=\lg \left(\overline{\mathbb{Q}}^{\prime}\right), \operatorname{rang}(f)=A$.

(3) If $A^{0} \subseteq \alpha$ and $A^{n+1}=A^{n} \cup\left\{A_{\alpha}: \alpha \in A^{n}\right\}$, then $\bigcup_{n<\omega} A^{n}$ is strongly $\overline{\mathbb{Q}}$-closed and $\bigcup\left\{\alpha+1: \alpha \in A^{0}\right\}=\bigcup\left\{\alpha+1: \alpha \in \bigcup_{n<\omega} A^{n}\right\}$.

(4) PAUT $(\overline{\mathbb{Q}})$ is closed under composition and inverse. Similarly, if $f_{l} \in$ $\operatorname{PAUT}\left(\overline{\mathbb{Q}}^{l}, \overline{\mathbb{Q}}^{l+1}\right)$ for $l=1,2$, then $f_{2} \circ f_{1} \in \operatorname{PAUT}\left(\overline{\mathbb{Q}}^{1}, \overline{\mathbb{Q}}^{3}\right)$ and $f_{i}^{-1} \in$ $\operatorname{PAUT}\left(\overline{\mathbb{Q}}^{2}, \overline{\mathbb{Q}}^{1}\right)$. 
(5) If $\overline{\mathbb{Q}} \in \mathcal{K}_{\kappa}^{s}$ and $A \subseteq \lg (\overline{\mathbb{Q}})$ is strongly $\overline{\mathbb{Q}}$-closed and $\bar{p} \in \mathbb{P}_{\lg (\overline{\mathbb{Q}})}^{\prime}$, then $p\left\lceil A \in \mathbb{P}_{A}^{\prime}\right.$ and $\mathbb{P}_{\lg (\overline{\mathbb{Q}})}=$ " $(p\lceil A) \leq p$ " and

$$
q \in \mathbb{P}_{A}^{\prime} \& q \leq_{\mathbb{P}_{\lg (\overline{\mathbb{Q}})}} p \Rightarrow q \leq_{\mathbb{P}_{\lg (\overline{\mathbb{Q}})} p\lceil A .}
$$

Proof. Straightforward.

3.7. Definition. We say $\overline{\mathcal{F}}$ witnesses $A$ for $\overline{\mathbb{Q}}, \kappa$ in $A^{\prime}$ (or for $\left(\overline{\mathbb{Q}}, \kappa, A^{\prime}\right)$; we may omit $A^{\prime}$ if it is the strong $\overline{\mathbb{Q}}$-closure of $A$ ) if:

(a) $\overline{\mathbb{Q}} \in \mathcal{K}_{\kappa}^{s}$.

(b) $A \subseteq \lg (\overline{\mathbb{Q}}), A \subseteq A^{\prime} \subseteq \lg (\overline{\mathbb{Q}}), A$ is $\overline{\mathbb{Q}}$-closed, $A^{\prime}$ is strongly $\overline{\mathbb{Q}}$-closed.

(c) $\overline{\mathcal{F}}=\left\langle\mathcal{F}_{\gamma}: \gamma<\omega_{1}\right\rangle, \mathcal{F}_{\gamma}$ decreasing with $\gamma$ (this just for notational simplicity).

(d) $\mathcal{F}_{\gamma} \subseteq \operatorname{PAUT}(\overline{\mathbb{Q}})$ for $\gamma<\omega_{1}$.

(e) If $f \in \mathcal{F}_{\gamma}$ then:

$(\alpha) \operatorname{rang}(f) \subseteq A$.

$(\beta)|\operatorname{dom}(f)|<\kappa$.

$(\gamma)(\forall \alpha \in \operatorname{dom}(f))(\exists \beta \in \operatorname{dom}(f))\left[f(\beta)=\beta \& \alpha \in \operatorname{scl}_{\overline{\mathbb{Q}}}(\{\beta\})\right]$.

$(\delta) \operatorname{dom}(f) \subseteq A^{\prime}$.

(f) If $f_{1} \in \mathcal{F}_{\gamma_{1}}, \gamma_{2}<\gamma_{1}<\omega_{1}$ and $C \subseteq A^{\prime}(\subseteq \lg (\overline{\mathbb{Q}}))$ has cardinality $<\kappa$, then for some $f_{2} \in \mathcal{F}_{\gamma_{2}}$ we have: $f_{1} \subseteq f_{2}$ and $C \subseteq \operatorname{dom}\left(f_{2}\right)$ and $C \cap A \subseteq \operatorname{rang}\left(f_{2}\right)$.

(g) If $C \subseteq A$ is such that $|C|<\kappa$, then $\operatorname{id}_{C} \in \bigcap_{\gamma<\omega_{1}} \mathcal{F}_{\gamma}$.

3.8. Observation. Let $\overline{\mathbb{Q}} \in \mathcal{K}_{\kappa}^{s}$. $\left(\mathbb{P}_{A}^{\prime}\right)^{\overline{\mathbb{Q}}}$

(1) If $\beta^{*} \leq \lg (\overline{\mathbb{Q}})$, then $\overline{\mathbb{Q}} \uparrow \beta^{*} \in \mathcal{K}_{\kappa}^{s}$, and for $A \subseteq \beta^{*}$ we have $\left(\mathbb{P}_{A}^{\prime}\right)^{\overline{\mathbb{Q}} \uparrow \beta^{*}}=$

(2) If $\overline{\mathcal{F}}$ witnesses $A$ for $\overline{\mathbb{Q}}, \kappa$ in $A^{\prime}$ and $A^{\prime \prime} \subseteq A^{\prime} \subseteq \lg (\overline{\mathbb{Q}}), A \subseteq A^{\prime \prime}, A^{\prime \prime}$ is strongly $\overline{\mathbb{Q}}$-closed, then $\overline{\mathcal{F}}$ witnesses $A$ for $\overline{\mathbb{Q}}, \kappa$ in $A^{\prime \prime}$.

(3) If $\overline{\mathcal{F}}$ witnesses $A$ for $\overline{\mathbb{Q}}, \kappa$ in $A^{\prime}$ and $\beta^{*} \leq \lg (\overline{\mathbb{Q}})$ and $A^{\prime \prime}$ is the strong $\overline{\mathbb{Q}}$-closure of $A \cap \beta^{*}$, then

$$
\left\langle\left\{f \uparrow \bigcup\left\{\operatorname{scl}_{\overline{\mathbb{Q}}}(\{\beta\}): \beta \in \operatorname{dom}(f) \cap \beta^{*}\right\}: f \in \mathcal{F}_{1+\gamma}\right\}: \gamma<\omega_{1}\right\rangle
$$

witnesses $A \cap \beta^{*}$ for $\overline{\mathbb{Q}}, \kappa$ in $A^{\prime \prime}$ (why $1+\gamma$ ? to preserve also "transitive closure of $\gamma_{1} \in A_{\gamma_{2}}$ "; see 3.11(1) below).

Proof. Straightforward.

3.9. Claim. Let $\overline{\mathbb{Q}} \in \mathcal{K}_{\kappa}^{s}$.

(1) If $\overline{\mathcal{F}}$ witnesses $A$ for $\overline{\mathbb{Q}}, \kappa$ inside $A^{\prime}=\operatorname{scl}_{\overline{\mathbb{Q}}}(A)$, then:

(a) $\mathbb{P}_{A}^{\prime} \lessdot \mathbb{P}_{\lg (\overline{\mathbb{Q}})}^{\prime}$ 
(b) $p, q \in \mathbb{P}_{A}^{\prime}$ are compatible in $\mathbb{P}_{\lg (\overline{\mathbb{Q}})}^{\prime}$ iff they are compatible in $\mathbb{P}_{A}^{\prime}$. Also if $p, q \in \mathbb{P}_{A}^{\prime}$ and $\gamma \in \operatorname{dom}(p) \cap \operatorname{dom}(q)$, then

$$
q\left\lceil\gamma \Vdash_{\mathbb{P}_{\gamma}^{\prime}} " p(\gamma) \leq \underset{\sim}{\mathbb{Q}_{\gamma}} q(\gamma) " \Rightarrow q\left\lceil\gamma \Vdash_{\mathbb{P}_{\gamma \cap A}^{\prime}} " p(\gamma) \leq \underset{\sim}{\mathbb{Q}_{\gamma}} q(\gamma) .\right.\right.
$$

(c) $\mathcal{I} \subseteq \mathbb{P}_{A}^{\prime}$ is predense in $\mathbb{P}_{\lg (\overline{\mathbb{Q}})}^{\prime}$ iff it is predense in $\mathbb{P}_{A}^{\prime}$ (without loss of generality $\mathcal{I}$ is countable $)$.

(d) There are $\left({ }^{5}\right)$ unique $f, \overline{\mathbb{Q}}^{\prime}$ such that $\overline{\mathbb{Q}}^{\prime} \in \mathcal{K}_{\kappa}^{s}, f \in \operatorname{PAUT}\left(\overline{\mathbb{Q}}^{\prime}, \overline{\mathbb{Q}}\right)$, $f$ is order preserving, $\operatorname{dom}(f)=\lg \left(\overline{\mathbb{Q}}^{\prime}\right)(=\operatorname{otp}(A))$ and $\operatorname{rang}(f)$

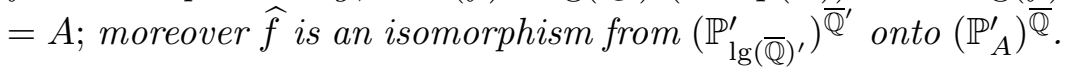

(2) Moreover:

(e) For every $p, q \in \mathbb{P}_{A^{\prime}}^{\prime}$ there is $\gamma<\omega_{1}$ such that:

(i) If $f \in \mathcal{F}_{\gamma}$ and $\operatorname{supp}(p) \cup \operatorname{supp}(q) \subseteq \operatorname{dom}(f)$, then $\mathbb{P}_{A^{\prime}}^{\prime}=$ " $p \leq q " \Leftrightarrow \mathbb{P}_{\lg \left(\overline{\mathbb{Q}^{\prime}}\right)}^{\prime} \models$ " $\widehat{f}(p) \leq \widehat{f}(q) "$.

(ii) If $f \in \mathcal{F}_{\gamma}$ and $\operatorname{supp}(p) \cup \operatorname{supp}(q) \subseteq \operatorname{rang}(f)$, then

$$
\mathbb{P}_{A^{\prime}}^{\prime} \models \text { " } \widehat{f}^{-1}(p) \leq \widehat{f}^{-1}(q) " \Leftrightarrow \mathbb{P}_{A^{\prime}}^{\prime} \models " p \leq q " .
$$

(f) For every $p, q \in \mathbb{P}_{A^{\prime}}^{\prime}$ there is $\gamma<\omega_{1}$ such that:

(i) If $f \in \mathcal{F}_{\gamma}$ and $\operatorname{supp}(p) \cup \operatorname{supp}(q) \subseteq \operatorname{dom}(f)$, then $p, q$ are compatible in $\mathbb{P}_{\lg (\overline{\mathbb{Q}})}^{\prime}$ iff $\widehat{f}(p), \widehat{f}(q)$ are compatible in $\mathbb{P}_{\lg \left(\overline{\mathbb{Q}}^{\prime}\right)}^{\prime}$.

(ii) If $f \in \mathcal{F}_{\gamma}$ and $\operatorname{supp}(p) \cup \operatorname{supp}(q) \subseteq \operatorname{rang}(f)\left(\right.$ so $\left.p, q \in \mathbb{P}_{A}^{\prime}\right)$, then $\widehat{f}^{-1}(p), \widehat{f}^{-1}(q)$ are compatible in $\mathbb{P}_{A^{\prime}}^{\prime}$ iff $p, q$ are compatible in $\mathbb{P}_{A^{\prime}}^{\prime}$.

(g) For every countable $\mathcal{I} \subseteq \mathbb{P}_{A^{\prime}}^{\prime}$, there is $\gamma<\omega_{1}$ such that:

(i) If $f \in \mathcal{F}_{\gamma}$ and $\bigcup_{p \in \mathcal{I}} \operatorname{supp}(p) \subseteq \operatorname{dom}(f)$, then $\mathcal{I}$ is predense in $\mathbb{P}_{A^{\prime}}^{\prime}$ iff $\widehat{f}(\mathcal{I})$ is predense in $\mathbb{P}_{A^{\prime}}^{\prime}$.

(ii) If $f \in \mathcal{F}_{\gamma}$ and $\bigcup_{p \in \mathcal{I}} \operatorname{supp}(p) \subseteq \operatorname{rang}(f)$ (so $\left.\mathcal{I} \subseteq \mathbb{P}_{A}^{\prime}\right)$, then $\left\{\widehat{f}^{-1}(p): p \in \mathcal{I}\right\}$ is predense in $\mathbb{P}_{A^{\prime}}^{\prime}$ iff $\mathcal{I}$ is predense in $\mathbb{P}_{A^{\prime}}^{\prime}$.

(h) For every Borel function

$$
\mathcal{B}=\mathcal{B}\left(\ldots, \text { truth value }\left(\xi_{n} \in \tau_{\alpha_{n}}\right), \ldots\right)_{n<\omega}
$$

from ${ }^{\omega} 2$ to $\mathbf{V}$ such that $\alpha_{n} \in A^{\prime}$ and $p \in \mathbb{P}_{A^{\prime}}^{\prime}$ there is $\gamma<\omega_{1}$ such that:

(i) If $f \in \mathcal{F}_{\gamma}$ and $\operatorname{supp}(p) \cup\left\{\alpha_{n}: n<\omega\right\} \subseteq \operatorname{dom}(f)$ and $x \in \mathbf{V}$, then $p \Vdash_{\mathbb{P}_{\lg (\mathbb{Q})}^{\prime}}$ "B $\mathcal{B}\left(\ldots, \text { truth value }\left(\xi_{n} \in \bar{\tau}_{\alpha_{n}}\right), \ldots\right)_{n<\omega}=x$ " iff $\widehat{f}(p) \Vdash_{\mathbb{P}_{\mathrm{g}(\overline{\mathbb{Q}})}^{\prime}}$ "B $\left(\ldots, \text { truth value }\left(\xi_{n} \in \tau_{f\left(\alpha_{n}\right)}\right), \ldots\right)_{n<\omega}=x "$.

$\left({ }^{5}\right)$ In fact, this does not depend on $A$ having a witness (but not necessarily the "moreover" part). 
(ii) If $f \in \mathcal{F}_{\gamma}$ and $\operatorname{supp}(p) \cup\left\{\alpha_{n}: n<\omega\right\} \subseteq \operatorname{rang}(f)$ and $x \in \mathbf{V}$, then $p \Vdash_{\mathbb{P}_{\lg (\mathbb{\Phi})}^{\prime}}$ “B $\left(\ldots \text {, truth value }\left(\xi_{n} \in \tau_{\alpha_{n}}\right), \ldots\right)_{n<\omega}=x$ " iff $\widehat{f}^{-1}(p) \Vdash_{\mathbb{P}_{\lg (\mathbb{Q})}^{\prime}}$ “B $\left(\ldots, \text { truth value }\left(\xi_{n} \in \mathcal{\tau}_{f^{-1}\left(\alpha_{n}\right)}\right), \ldots\right)_{n<\omega}=x "$.

Proof. We prove $\left((1)+(2)\right.$ together) by induction on $\alpha_{0}^{*}=\alpha^{*}=\lg (\overline{\mathbb{Q}})$. Arriving at $\alpha^{*}$, we note various implications:

$(*)_{1} \quad$ For $\alpha<\alpha^{*}$, if we replace $A, A^{\prime}$ and $\overline{\mathcal{F}}$ by $A \cap \alpha, A^{\prime \prime}$ (any strong $\overline{\mathbb{Q}}$-closed set such that $\left.A \cap \alpha \subseteq A^{\prime \prime} \subseteq A^{\prime} \cap \alpha\right)$ and $\overline{\mathcal{F}} 1 A^{\prime \prime}:=\langle\{f\lceil B$ : $B \subseteq \operatorname{dom}(f) \cap A^{\prime \prime}$ and $(\forall \alpha \in B)(\exists \beta \in B)\left[f(\beta)=\beta \& \alpha \in \operatorname{scl}_{\overline{\mathbb{Q}}}(\{\beta\}):\right.$ $\left.\left.f \in \mathcal{F}_{i}\right\}: i<\omega_{1}\right\rangle$ respectively, Claim 3.9 holds.

$(*)_{2} \quad$ Clause (d) (for $\alpha^{*}$ ) follows (from the induction hypothesis).

$(*)_{3}$ If clause (e) holds, then clause (b) holds.

[Why? The second phrase in clause (b) holds by clause (e)(ii) as $C \subseteq A$ \& $|C| \leq \aleph_{0} \& i<\omega_{1} \Rightarrow \operatorname{id}_{C} \in \mathcal{F}_{i}$. If $p, q$ are compatible in $\mathbb{P}_{A}^{\prime}$, then they have a common upper bound there, which "works" in $\mathbb{P}_{\lg (\overline{\mathbb{Q}})}^{\prime}$, too, by the previous sentence. Assume $p, q$ have a common upper bound $r$ in $\mathbb{P}_{\lg (\overline{\mathbb{Q}})}^{\prime}$, so by 3.6(5) also $r^{\prime}:=r\left\lceil A^{\prime} \in \mathbb{P}_{A^{\prime}}^{\prime}\right.$ is a common upper bound of $p$ and $q$.

Let $\gamma_{1}, \gamma_{2}<\omega_{1}$ be as guaranteed in clause (e) for $p \leq r^{\prime}, q \leq r^{\prime}$ respectively and let $\gamma=\max \left\{\gamma_{1}, \gamma_{2}\right\}$ and $f=\operatorname{id}_{\operatorname{supp}(p) \cup \operatorname{supp}(q)}$; now $f \in \mathcal{F}_{\gamma+1}$ by clause (g), Definition 3.7 and there is $f^{\prime}$ with $f \subseteq f^{\prime} \in \mathcal{F}_{\gamma}$ such that $\operatorname{supp}\left(r^{\prime}\right) \subseteq \operatorname{dom}\left(f^{\prime}\right)$.

So $\widehat{f}^{\prime}(p)=\widehat{f}(p)=p, \widehat{f}^{\prime}(q)=\widehat{f}(q)=q, \widehat{f}^{\prime}\left(r^{\prime}\right) \in \mathbb{P}_{A}^{\prime}$, and by clause (e) we have $p=\widehat{f}^{\prime}(p) \leq \widehat{f}^{\prime}\left(r^{\prime}\right) \in \mathbb{P}_{A}^{\prime}, q=\widehat{f}^{\prime}(q) \leq \widehat{f}^{\prime}\left(r^{\prime}\right) \in \mathbb{P}_{A}^{\prime}$ and we are done.]

$(*)_{4} \quad$ If clauses (e),(f) hold then clause (c) holds.

[Why? If $\mathcal{I}$ is not predense in $\mathbb{P}_{A}^{\prime}$ then there is $q \in \mathbb{P}_{A}^{\prime}$ incompatible in $\mathbb{P}_{A}^{\prime}$ with every $p \in \mathcal{I}$, hence by clause (b) which holds by $(*)_{3}, q$ is incompatible with $p$ in $\mathbb{P}_{\lg (\overline{\mathbb{Q}})}^{\prime}$, hence $\mathcal{I}$ is not predense in $\mathbb{P}_{\lg (\overline{\mathbb{Q}})}^{\prime}$. Next assume $\mathcal{I}$ is predense in $\mathbb{P}_{A}^{\prime}$, and let $\mathcal{J}$ be a maximal antichain of $\mathbb{P}_{A}^{\prime}$ of elements above some member of $\mathcal{I}$; so by clause (b) (which holds by $\left.(*)_{3}\right), \mathcal{J}$ is an antichain in $\mathbb{P}_{\lg (\overline{\mathbb{Q}})}^{\prime}$, hence is countable.

Let $q$ be any member of $\mathbb{P}_{A^{\prime}}^{\prime}$; let $\gamma=\sup \{\gamma(p, q): p \in \mathcal{I}\}$ with $\gamma(p, q)$ as in clause (f).

Now let $C=\bigcup_{p \in \mathcal{J}} \operatorname{supp}(p)$; it is a countable subset of $A$, hence $f_{0}=$ $\operatorname{id}_{C} \in \mathcal{F}_{\gamma+1}$, so there is $f$ with $f_{0} \subseteq f \in \mathcal{F}_{\gamma}$ such that $\operatorname{supp}(q) \subseteq \operatorname{dom}(f)$. Now $\gamma \geq \gamma(p, q)$ for $p \in \mathcal{J}$, hence

$(\forall p \in \mathcal{J})\left(p, q\right.$ are compatible in $\mathbb{P}_{A^{\prime}}$ iff $\widehat{f}(p), \widehat{f}(q)$ are compatible in $\left.\mathbb{P}_{\lg (\overline{\mathbb{Q}})}^{\prime}\right)$. 
As $\widehat{f}(q) \in \mathbb{P}_{A}^{\prime}$ and $\mathcal{J}$ is a maximal antichain of $\mathbb{P}_{A}^{\prime}$, for some $p \in \mathcal{J}, \widehat{f}(q), p$ are compatible in $\mathbb{P}_{A}^{\prime}$, hence in $\mathbb{P}_{A^{\prime}}^{\prime}$. But $f(p)=p$, so $q, p$ are compatible in $\mathbb{P}_{\lg (\overline{\mathbb{Q}})}^{\prime}$; but by the choice of $\mathcal{J}$ there is $p^{\prime} \in \mathcal{I}$ with $p^{\prime} \leq p$, so $q$ is compatible with some member of $\mathcal{J}$, i.e. with $p^{\prime}$. As $q \in \mathbb{P}_{A^{\prime}}^{\prime}$ was arbitrary, this proves that $\mathcal{I}$ is predense in $\mathbb{P}_{A^{\prime}}^{\prime}$, completing the second implication in the proof of $\left.(*)_{4} \cdot\right]$

$(*)_{5} \quad$ If clauses (e), (f) hold then clause (a) holds.

[Why? By $(*)_{3}+(*)_{4}$.]

$(*)_{6} \quad$ If clauses (e), (f), (g) hold for $\alpha^{*}$, then clause (h) holds.

[Why? First, it is enough to deal with the case where the range of the Borel function is $\{0,1\}$. Now we prove the assertion by induction on the depth of the Borel function.

CASE A: $\mathcal{B}$ is atomic, i.e. $\mathcal{B}$ is the truth value of $\xi \in \mathcal{\tau}_{\gamma}$ for some $\xi<\mu_{\gamma}$. Clearly $\gamma<\alpha^{*}$ so we can apply the induction hypothesis to $\gamma$. So $p \Vdash$ "B $\left(\xi \in \mathcal{\tau}_{\gamma}\right)=i$ ", where $p \in \mathbb{P}_{A^{\prime}}^{\prime}$ is equivalent to $p\lceil\gamma \Vdash$ " $p(\gamma)=$ truth". Now $p(\gamma)$ has the form $\mathcal{B}^{\prime}\left(\ldots \text {, truth value }\left(\xi_{n} \in \tau_{\beta_{n}}\right), \ldots\right)_{n<\omega}$, where $\beta_{n} \in A_{\gamma}$, $\xi_{n}<\mu_{\beta_{n}}, \mathcal{B}^{\prime} \in \mathbf{V}$ a Borel function, so the statement is equivalent to $p\lceil\gamma \Vdash$ " $\mathcal{B}^{\prime}\left(\ldots, \text { truth value }\left(\xi_{n} \in \tau_{\beta_{n}}\right), \ldots\right)_{n<\omega}=$ truth. As $A^{\prime}$ is strongly $\overline{\mathbb{Q}}$-closed, clearly $\beta_{n} \in A_{\gamma} \subseteq A^{\prime}$, so we can apply the induction hypothesis on $\gamma$ using clause $(\mathrm{h})$ there.

CASE B: $\mathcal{B}=\neg \mathcal{B}^{\prime}$ (i.e. $1-\mathcal{B}$ ). By the way we phrase the statement, it follows from the statement on $\mathcal{B}^{\prime}$.

CAse C: $\mathcal{B}=\bigwedge_{n<\omega} \mathcal{B}_{n}$. Let $\mathcal{I}$ be a maximal subset of

$\left\{q \in \mathbb{P}_{A^{\prime}}^{\prime}:\right.$ (i) $q$ forces $\mathcal{B}_{n}$ for every $n$ or for some $n, q$ forces $\neg \mathcal{B}_{n}$,

(ii) $p \leq q$ or $q, p$ are incompatible $\}$,

which is an antichain in $\mathbb{P}_{\lg (\overline{\mathbb{Q}})}$. Let $\gamma_{\mathcal{I}}<\omega_{1}$ be as guaranteed by $3.9(\mathrm{~g})$, and for $q \in \mathcal{I}$ let $\gamma(p, q)<\omega_{1}$ be as guaranteed by 3.9(f) if $p, q$ are incompatible, and by $3.9(\mathrm{e})$ if $p \leq q$.

For each $q \in \mathcal{I}$ let $\gamma_{n}(q)$ be the $\gamma$ guaranteed for $q, \mathcal{B}_{n}$ for both $x=0$ and $x=1$. For $q_{1} \neq q_{2}$ from $\mathcal{I}$ (so incompatible) let $\gamma\left(q_{1}, q_{2}\right)<\omega_{1}$ be as guaranteed in clause (f). Let $\gamma^{*}=\sup \left(\left\{\gamma_{\mathcal{I}}\right\} \cup\{\gamma(p, q): q \in \mathcal{I}\} \cup\left\{\gamma_{n}(q)\right.\right.$ : $q \in \mathcal{I}, n<\omega\} \cup\left\{\gamma\left(q_{1}, q_{2}\right): q_{1} \neq q_{2}\right.$ from $\left.\left.\mathcal{I}\right\}\right)+1$.

Suppose $f_{1} \in \mathcal{F}_{\gamma^{*}}, \operatorname{supp}(p) \cup\left\{\alpha_{n}: n<\omega\right\} \subseteq \operatorname{dom}\left(f_{1}\right)$, where the $\alpha_{n}$ 's are from the statement of clause (h). We can find $f_{2}$ with $f_{1} \subseteq f_{2} \in \mathcal{F}_{\gamma^{*}-1}$ and $\bigcup_{q \in \mathcal{I}} \operatorname{dom}(q) \subseteq \operatorname{dom}\left(f_{2}\right)$.

Let $\mathcal{I}_{0}=\{q: p \leq q \in \mathcal{I}\}$ and $\mathcal{I}_{1}=\mathcal{I} \backslash \mathcal{I}_{0}$. Now $q_{1} \neq q_{2} \in \mathcal{I} \Rightarrow \gamma\left(q_{1}, q_{2}\right) \leq$ $\gamma^{*}-1$ and $\gamma_{\mathcal{I}} \leq \gamma^{*}-1$, hence $\mathcal{I}^{\prime}=\left\{\widehat{f}_{2}(q): q \in \mathcal{I}\right\}$ is a maximal antichain. 
Also $\gamma(p, q) \leq \gamma_{1}$, hence

$$
\begin{gathered}
q \in \mathcal{I}_{0}^{\prime}:=\left\{\widehat{f_{2}}(q): q \in \mathcal{I}_{0}\right\} \Rightarrow \widehat{f}_{2}(p) \leq q, \\
q \in \mathcal{I}_{1}^{\prime}=\left\{\widehat{f}_{1}(q): q \in \mathcal{I}_{1}\right\} \Rightarrow \widehat{f_{2}}(p), q \text { incompatible. }
\end{gathered}
$$

Also for $q \in \mathcal{I}_{0}$,

$$
\begin{aligned}
& q \Vdash " \mathcal{B}_{n}=0 " \Leftrightarrow f_{2}(q) \Vdash “ f_{2}\left(\mathcal{B}_{n}\right)=0 ", \\
& q \Vdash " \mathcal{B}_{n}=1 " \Leftrightarrow f_{2}(q) \Vdash " f_{2}\left(\mathcal{B}_{n}\right)=1 . "
\end{aligned}
$$

The rest should be clear.]

So together it is enough to prove clauses (e), (f), (g).

$(*)_{7} \quad$ Clause (e) holds.

If $\operatorname{dom}(p)=\emptyset$ this is trivial so suppose not, and if $\operatorname{dom}(p) \nsubseteq \mathbb{\operatorname { d o m }}(q)$ the equivalence is trivial so assume $\emptyset \neq \operatorname{dom}(p) \subseteq \operatorname{dom}(q)$. Let $\alpha^{\otimes}=$ $\max (\operatorname{dom}(q))$. Now if $\alpha^{\otimes}+1<\alpha^{*}$ we can use the induction hypothesis on $\gamma^{*}+1$, so assume $\alpha^{*}=\alpha^{\otimes}+1$ (we can also discard the case $\gamma^{*} \notin \operatorname{dom}(p)$ if we like).

Now $\mathbb{P}_{A^{\prime}}^{\prime}=$ " $p \leq q$ " iff $(\alpha)+(\beta)$, where:

( $\alpha) \mathbb{P}_{A^{\prime} \cap \gamma^{*}}^{\prime}=" p\left\lceil\alpha^{\otimes} \leq q\left\lceil\alpha^{\otimes "}\right.\right.$.

( $\beta) q \Vdash " p\left(\alpha^{\otimes}\right) \leq q "$.

Now for $(\alpha)$ get $\gamma_{1}$ by applying clause (e) to $\overline{\mathbb{Q}}\left\lceil\alpha^{\otimes}\right.$, and for $(\beta)$ get $\gamma_{2}$ by applying clause (h) to $\overline{\mathbb{Q}}\left\lceil\alpha^{\otimes}\right.$, so $\gamma=\max \left\{\gamma_{1}, \gamma_{2}\right\}$ is as required.

$(*)_{8} \quad$ Clause (f) holds.

As in the proof of clause (e), without loss of generality $\alpha^{*}=\alpha^{*}+1$ and $\alpha^{\otimes}=\max (\operatorname{dom}(p))=\max (\operatorname{dom}(q))$. Let $\left\{r_{n}: n<\omega\right\} \subseteq \mathbb{P}_{A_{\alpha} \otimes}^{\prime}$ be a maximal antichain such that each $r_{n}$ satisfies:

$(\alpha)$ It forces a truth value, say $\mathbf{t}_{n}$, to " $p\left(\alpha^{\otimes}\right), q\left(\alpha^{\otimes}\right)$ are compatible in $\mathbb{Q}_{\alpha} \otimes$.

( $\beta) r_{n} \geq p$ or $r_{n}, p$ are incompatible.

$(\gamma) r_{n} \geq q$ or $r_{n}, q$ are incompatible.

By applying clause (h) to $\overline{\mathbb{Q}}\left\lceil\alpha^{\otimes}\right.$, without loss of generality $\left\{r_{n}: n<\omega\right\}$ $\subseteq \mathbb{P}_{A}^{\prime}$.

Now clearly $p \leq q$ iff $\bigvee_{n}\left[p \leq r_{n} \& q \leq r_{n} \& \mathbf{t}_{n}=\right.$ truth $]$, and we can apply the induction hypothesis to each of those countably many statements.

$(*)_{9} \quad$ Clause (g) holds.

Without loss of generality $\mathcal{I}$ is countable. Now if $w=\bigcup\{\operatorname{dom}(p): p \in \mathcal{I}\}$ has no last element, clearly $\mathcal{I}$ is predense iff $\alpha \in w \Rightarrow \mathcal{I}^{[\alpha]}=\{p\lceil\alpha: p \in \mathcal{I}\}$ is predense; so we can finish by the induction hypothesis. So assume $\alpha^{\otimes}$ is the last element in $w$. Let $\left\{r_{n}: n<\omega\right\} \subseteq \mathbb{P}_{A^{\prime} \cap \alpha^{\otimes}}^{\prime}$ be a maximal antichain 
of it, each $r_{n}$ forcing a truth value to " $\left\{p\left(\alpha^{\otimes}\right): p\left\lceil\alpha^{\otimes} \in G_{\mathbb{P}_{\alpha \otimes}}\right\}\right.$ is a maximal antichain of $\mathbb{Q}_{\alpha}$ ". 3.9

3.10. Definition. (1) We say $\overline{\mathcal{F}}$ is a $\kappa$-witness for $\left(\overline{\mathbb{Q}}^{1}, \overline{\mathbb{Q}}^{2}\right)$ if:

(a) $\overline{\mathbb{Q}}^{1} \in \mathcal{K}_{\kappa}^{s}$ and $\overline{\mathbb{Q}}^{2} \in \mathcal{K}_{\kappa}^{s}$ (so $\kappa>\aleph_{0}$ ).

(b) $\overline{\mathcal{F}}=\left\langle\mathcal{F}_{\gamma}: \gamma<\omega_{1}\right\rangle$ and $\mathcal{F}_{\gamma}$ is decreasing with $\gamma$ (for notational simplicity).

(c) $\mathcal{F}_{\gamma} \subseteq \operatorname{PAUT}\left(\overline{\mathbb{Q}}^{1}, \overline{\mathbb{Q}}^{2}\right)$ for $\gamma<\omega_{1}$.

(d) If $f \in \mathcal{F}_{\gamma}$, then $\operatorname{dom}(f) \subseteq \lg \left(\overline{\mathbb{Q}}^{1}\right)$ and $\operatorname{rang}(f) \subseteq \lg \left(\overline{\mathbb{Q}}^{2}\right)$.

(e) If $f_{1} \in \mathcal{F}_{\gamma_{1}}, \gamma_{2}<\gamma_{1}<\omega_{1}, C_{1} \subseteq \lg \left(\overline{\mathbb{Q}}^{1}\right), C_{2} \subseteq \lg \left(\overline{\mathbb{Q}}^{2}\right),\left|C_{1}\right|<\kappa$, $\left|C_{2}\right|<\kappa$, then for some $f_{2} \in \mathcal{F}_{\gamma_{2}}$ we have

$$
f_{1} \subseteq f_{2}, \quad C_{1} \subseteq \operatorname{dom}\left(f_{2}\right), \quad C_{2} \subseteq \operatorname{rang}\left(f_{2}\right) .
$$

(2) We say $\overline{\mathcal{F}}$ is an explicit $\kappa$-witness for $\left(\overline{\mathbb{Q}}^{1}, \overline{\mathbb{Q}}^{2}\right)$ if (a)-(d) above hold and:

$(\mathrm{e})^{\prime}$ If $f_{1} \in \mathcal{F}_{\gamma_{1}}, \gamma_{2}<\gamma_{1}<\omega_{1}, C_{1} \subseteq \lg \left(\overline{\mathbb{Q}}^{1}\right) \cap \operatorname{scl}_{\overline{\mathbb{Q}}^{1}}\left(\operatorname{dom}\left(f_{1}\right)\right), C_{2} \subseteq$ $\lg \left(\overline{\mathbb{Q}}^{2}\right) \cap \operatorname{scl}_{\overline{\mathbb{Q}}^{2}}\left(\operatorname{dom}\left(f_{2}\right)\right),\left|C_{1}\right|<\kappa,\left|C_{2}\right|<\kappa$, then for some $f_{2} \in$ $\mathcal{F}_{\gamma_{2}}$ we have $f_{1} \subseteq f_{2}, C_{1} \subseteq \operatorname{dom}\left(f_{2}\right), C_{2} \subseteq \operatorname{rang}\left(f_{2}\right), \operatorname{dom}\left(f_{2}\right) \subseteq$ $\operatorname{scl}_{\overline{\mathbb{Q}}^{1}}\left(\operatorname{dom}\left(f_{1}\right)\right), \operatorname{rang}\left(f_{2}\right) \subseteq \operatorname{scl}_{\overline{\mathbb{Q}}^{2}}\left(\operatorname{rang}\left(f_{1}\right)\right)$.

(f) For $f \in \mathcal{F}_{\gamma}, g \subseteq f \Rightarrow g \in \mathcal{F}_{\gamma}$.

(g) If $f \in \mathcal{F}_{\gamma}$ and $\alpha, \beta \in \operatorname{dom}(f)$, then

$$
\alpha \in \operatorname{scl}_{\overline{\mathbb{Q}}^{1}}(\{\beta\}) \Leftrightarrow f(\alpha) \in \operatorname{scl}_{\overline{\mathbb{Q}}^{2}}(\{f(\beta)\}) .
$$

(h) If $C_{1} \subseteq \lg \left(\overline{\mathbb{Q}}^{1}\right)$ and $C_{2} \subseteq \lg \left(\overline{\mathbb{Q}}^{2}\right)$ are countable and $\gamma<\omega_{1}$, then for some $f \in \mathcal{F}_{\gamma}$ we have $C_{1} \subseteq \operatorname{dom}(f)$ and $C_{2} \subseteq \operatorname{rang}(f)$.

3.11. Claim. Assume $\overline{\mathcal{F}}$ is a $\kappa$-witness for $\left(\overline{\mathbb{Q}}^{1}, \overline{\mathbb{Q}}^{2}\right)$.

(1) If $\gamma \in\left(0, \omega_{1}\right)$ and $f \in \mathcal{F}_{\gamma}$ and $\alpha, \beta<\lg \left(\overline{\mathbb{Q}}^{1}\right)$, then

$$
\alpha \in \operatorname{scl}_{\overline{\mathbb{Q}}^{1}}(\{\beta\}) \Leftrightarrow f(\alpha) \in \operatorname{scl}_{\overline{\mathbb{Q}}^{2}}(\{f(\beta)\}) .
$$

(2) Let $\overline{\mathcal{F}}^{-1}=\left\langle\mathcal{F}_{\gamma}^{-1}: \gamma<\omega_{1}\right\rangle$ and $\mathcal{F}_{\gamma}^{-1}=\left\{f^{-1}: f \in \mathcal{F}_{\gamma}\right\}$. Then $\overline{\mathcal{F}}^{-1}$ is a $\kappa$-witness for $\left(\overline{\mathbb{Q}}^{2}, \overline{\mathbb{Q}}^{1}\right)$.

(3) If $A_{l} \subseteq \lg \left(\overline{\mathbb{Q}}^{l}\right)$ and $A_{l}=\operatorname{scl}_{\overline{\mathbb{Q}}^{l}}\left(A_{l}\right)$ for $l=1,2$ and we let $\mathcal{F}_{\gamma}^{\prime}=$ $\left\{f \cap X: f \in \mathcal{F}_{1+\gamma}, X \subseteq A_{1} \times A_{2}\right\}$, then $\left\langle\mathcal{F}_{\gamma}^{\prime}: \gamma<\omega_{1}\right\rangle$ is a $\kappa$-witness for $\left(\overline{\mathbb{Q}}^{1} \uparrow A_{1}, \overline{\mathbb{Q}}^{2} \uparrow A_{2}\right)$ and an explicit $\kappa$-witness for $i$; note that by renaming $A_{l}$ can become an ordinal.

(4) If $p \in\left(\mathbb{P}_{\lg \left(\overline{\mathbb{Q}}^{1}\right)}^{1}\right)^{\prime}, q \in\left(\mathbb{P}_{\lg \left(\overline{\mathbb{Q}}^{1}\right)}^{1}\right)^{\prime}$ and $\mathcal{I} \subseteq\left(\mathbb{P}_{\lg \left(\overline{\mathbb{Q}}^{1}\right)}^{1}\right)^{\prime}$ is countable, then for some $\gamma<\omega_{1}$ and every $f \in \mathcal{F}_{\gamma}$ we have:

( $\alpha)$ If $\operatorname{supp}(p) \cup \operatorname{supp}(q) \subseteq \operatorname{dom}(f)$, then

$$
\left(\mathbb{P}_{\lg \left(\overline{\mathbb{Q}}^{1}\right)}^{1}\right)^{\prime}=p \leq q \quad \text { iff } \quad\left(\mathbb{P}_{\lg \left(\overline{\mathbb{Q}}^{2}\right)}^{2}\right)^{\prime}=\widehat{f}(p) \leq \widehat{f}(q) .
$$


$(\beta)$ If $\operatorname{supp}(p) \cup \operatorname{supp}(q) \subseteq \operatorname{dom}(f)$, then $p, q$ are compatible in $\left(\mathbb{P}_{\lg \left(\overline{\mathbb{Q}}^{1}\right)}^{1}\right)^{\prime}$ iff $\widehat{f}(p), \widehat{f}(q)$ are incompatible in $\left(\mathbb{P}_{\lg \left(\overline{\mathbb{Q}}^{1}\right)}^{2}\right)^{\prime}$.

$(\gamma)$ If $\bigcup_{r \in \mathcal{I}} \operatorname{supp}(r) \subseteq \operatorname{dom}(f)$, then $\mathcal{I}$ is predense in $\left(\mathbb{P}_{\lg \left(\overline{\mathbb{Q}}^{1}\right)}^{1}\right)^{\prime}$ iff $\widehat{f}(\mathcal{I})=\{\widehat{f}(r): r \in \mathcal{I}\}$ is predense in $\left(\mathbb{P}_{\lg \left(\overline{\mathbb{Q}}^{2}\right)}^{2}\right)^{\prime}$.

(5) For every Borel function $\mathcal{B}=\mathcal{B}\left(\ldots, \mathbf{t}_{n}, \ldots\right)_{n \in \omega}\left(\right.$ for $\mathbf{t}_{n}$ a truth value, with values in $\mathbf{V})$ and $\left\langle\left(\xi_{n}, \alpha_{n}\right): n<\omega\right\rangle, \alpha_{n}<\lg \left(\overline{\mathbb{Q}}^{1}\right)$, and $p \in\left(\mathbb{P}_{\lg \left(\overline{\mathbb{Q}}^{1}\right)}^{1}\right)^{\prime}$ there is $\gamma<\omega_{1}$ such that if $f \in \mathcal{F}_{\gamma},\left\{\alpha_{n}: n<\omega\right\} \cup \operatorname{supp}(p) \subseteq \operatorname{dom}(f)$ and $x \in \mathbf{V}$, then

$$
\begin{gathered}
p \Vdash_{\mathbb{P}_{\lg \left(\mathbb{Q}^{1}\right)}^{1}} \text { " } \mathcal{B}\left(\ldots, \text { truth value }\left(\xi_{n} \in \mathcal{\tau}_{\alpha_{n}}\right), \ldots\right)_{n<\omega}=x " \quad \text { iff } \\
\widehat{f}(p) \Vdash_{\mathbb{P}_{\lg \left(\overline{\mathbb{Q}}^{2}\right)}^{2}} \text { " } \mathcal{B}\left(\ldots, \text { truth value }\left(\xi_{n} \in \mathcal{\tau}_{f}\left(\alpha_{n}\right), \ldots\right)_{n<\omega}=x " .\right.
\end{gathered}
$$

Proof. (1), (2), (3) Easy.

(4), (5) It suffices to prove 3.12 below.

3.12. Claim. If $\overline{\mathcal{F}}$ is an explicit $\kappa$-witness for $\left(\overline{\mathbb{Q}}^{1}, \overline{\mathbb{Q}}^{2}\right)$ then $(4),(5)$ of 3.11 hold.

Proof. We prove this by induction on $\alpha^{*}=\max \left\{\lg \left(\overline{\mathbb{Q}}^{1}\right), \lg \left(\overline{\mathbb{Q}}^{2}\right)\right\}$, and for a fixed $\alpha^{*}$ by induction on $\beta^{*}=\min \left\{\lg \left(\overline{\mathbb{Q}}^{1}\right), \lg \left(\overline{\mathbb{Q}}^{2}\right)\right\}$.

As above it is enough to prove part (4). This is done by cases. Without loss of generality $A^{\prime}$ is the strong $\overline{\mathbb{Q}}$-closure of $A$.

Case 1: $\lg \left(\overline{\mathbb{Q}}^{1}\right)=0$. Trivial.

CASE 2: $\operatorname{cf}\left(\lg \left(\overline{\mathbb{Q}}^{1}\right)\right)>\aleph_{0}$. So let $p, q, \mathcal{I}$ be given and choose $\alpha^{1}<\lg \left(\overline{\mathbb{Q}}^{1}\right)$ such that $p, q \in\left(\mathbb{P}_{\alpha^{1}}^{1}\right)^{\prime}$ and $\mathcal{I} \subseteq\left(\mathbb{P}_{\alpha^{1}}^{1}\right)^{\prime}$. Let $\overline{\mathbb{Q}}^{0}=\overline{\mathbb{Q}}^{1}\left\lceil\alpha^{1}, \mathcal{F}_{\gamma}^{\prime}=\mathcal{F}_{\gamma} \cap\left(\alpha^{1} \times\right.\right.$ $\left.\lg \left(\overline{\mathbb{Q}}^{2}\right)\right)$, and apply the induction hypothesis to $\overline{\mathbb{Q}}^{0}, \overline{\mathbb{Q}}^{2},\left\langle\mathcal{F}_{\gamma}^{\prime}: \gamma<\omega_{1}\right\rangle$ and to $p, q, \mathcal{I}$ and get $\gamma<\omega_{1}$; we shall prove that it works for $\overline{\mathbb{Q}}^{1}, \overline{\mathbb{Q}}^{2}, \overline{\mathcal{F}}, p, q, \mathcal{I}$. So let $f \in \mathcal{F}_{\gamma}$ be such that $\operatorname{supp}(p) \cup \operatorname{supp}(q) \subseteq \operatorname{dom}(f)$ (needed if we are dealing with clauses $(\alpha)$ or $(\beta)$ of $3.11(4))$ and such that $\bigcup_{r \in \mathcal{I}} \operatorname{supp}(r) \subseteq \operatorname{dom}(f)$ (needed if we are dealing with clause $(\gamma)$ of $3.11(4)$ ). Now $f^{\prime}:=f\left\lceil\alpha^{1} \in \mathcal{F}_{\gamma}^{\prime}\right.$, so:

$(*)_{1} \quad\left(\mathbb{P}_{\alpha^{1}}^{1}\right)^{\prime}=" p \leq q " \Leftrightarrow\left(\mathbb{P}_{\lg \left(\overline{\mathbb{Q}}^{2}\right)}^{2}\right)^{\prime}=" \widehat{f}^{\prime}(p) \leq \widehat{f}^{\prime}(q) "$.

$(*)_{2} \quad p, q$ are compatible in $\mathbb{P}_{\alpha^{1}}^{1}$ iff $\widehat{f}^{\prime}(p), \widehat{f}^{\prime}(q)$ are compatible in $\left(\mathbb{P}_{\lg \left(\overline{\mathbb{Q}}^{2}\right)}^{2}\right)^{\prime}$.

$(*)_{3} \quad \mathcal{I}$ is predense in $\left(\mathbb{P}_{\alpha^{1}}^{1}\right)^{\prime}$ iff $\left\{\widehat{f}^{\prime}(r): r \in \mathcal{I}\right\}$ is predense in $\left(\mathbb{P}_{\lg \left(\overline{\mathbb{Q}}^{2}\right)}^{2}\right)^{\prime}$.

As in all three cases we can replace $\left(\mathbb{P}_{\alpha^{1}}^{1}\right)^{\prime}$ by $\left(\mathbb{P}_{\lg \left(\overline{\mathbb{Q}}^{1}\right)}^{1}\right)^{\prime}$ and $\widehat{f}^{\prime}$ by $\widehat{f}$ we are done.

CASE 3: $\operatorname{cf}\left(\lg \left(\overline{\mathbb{Q}}^{1}\right)\right)=\aleph_{0}$. Concerning clauses $(\alpha),(\beta)$ of $3.11(4)$ the proof is just as in case 2 , so we deal with clause $(\gamma)$. Let $\mathcal{I} \subseteq\left(\mathbb{P}_{\lg \left(\overline{\mathbb{Q}}^{1}\right)}^{1}\right)^{\prime}$ be 
countable. We choose $\alpha^{n}<\lg \left(\overline{\mathbb{Q}}^{1}\right)$ such that $\alpha^{n}<\alpha^{n+1}$ and $\bigcup_{n<\omega} \alpha^{n}=$ $\lg \left(\overline{\mathbb{Q}}^{1}\right)$ and let $\mathcal{I}_{n}=\left\{r\left\lceil\alpha^{n}: r \in \mathcal{I}_{n}\right\}\right.$ and $\mathcal{F}_{\gamma}^{n}=\mathcal{F}_{\gamma} \cap\left(\alpha^{n} \times \lg \left(\overline{\mathbb{Q}}^{2}\right)\right), \overline{\mathcal{F}}^{n}=$ $\left\langle\mathcal{F}_{\gamma}^{n}: \gamma<\omega_{1}\right\rangle$. For each $n$ apply the induction hypothesis to $\overline{\mathbb{Q}}^{1} \mid \alpha^{n}, \overline{\mathbb{Q}}^{2}, \overline{\mathcal{F}}^{n}$, $\mathcal{I}_{n}$ and get $\gamma_{n}<\omega_{1}$, and let $\gamma^{*}=\left(\bigcup_{n<\omega} \gamma_{n}\right)+1$. So let $f \in \mathcal{F}_{\gamma^{*}}$ be such that $\bigcup_{r \in \mathcal{I}} \operatorname{supp}(r) \subseteq \operatorname{dom}(f)$. First assume $\mathcal{I}$ is predense in $\left(\mathbb{P}_{\lg \left(\overline{\mathbb{Q}}^{1}\right)}^{1}\right)^{\prime}$.

So there is $q \in\left(\mathbb{P}_{\lg \left(\overline{\mathbb{Q}}^{1}\right)}^{1}\right)^{\prime}$ incompatible with every $r \in \mathcal{I}$, hence $q \in\left(\mathbb{P}_{\alpha_{n}}^{1}\right)^{\prime}$ for some $n$, so $q$ is incompatible with every $r \in \mathcal{I}_{n}$ in $\left(\mathbb{P}_{\alpha_{n}}^{1}\right)^{\prime}$, hence $\mathcal{I}_{n}$ is not predense in $\left(\mathbb{P}_{\alpha_{n}}^{1}\right)^{\prime}$. As $\gamma^{*}>\gamma_{n}$, necessarily $\left\{\widehat{f}(r): r \in \mathcal{I}_{n}\right\}$ is not predense in $\left(\mathbb{P}_{\lg \left(\overline{\mathbb{Q}}^{2}\right)}^{2}\right)^{\prime}$, which means that some $q^{\prime} \in\left(\mathbb{P}_{\lg \left(\overline{\mathbb{Q}}^{2}\right)}^{1}\right)^{\prime}$ is incompatible with $\widehat{f}(r)$ for every $r \in \mathcal{I}_{n}$. Now trivially

$$
r \in \mathcal{I} \Rightarrow \widehat{f}\left(r \mid \alpha_{n}\right) \leq_{\left(\mathbb{P}_{\lg \left(\overline{\mathbb{Q}}^{2}\right)}^{2}\right)^{\prime}} \widehat{f}(r)
$$

(look at the definition and note $r\left\lceil\alpha_{n} \leq r\right.$ and increasing $\gamma^{*}$ ), clearly $q^{\prime} \in$ $\left(\mathbb{P}_{\lg \left(\overline{\mathbb{Q}}^{2}\right)}^{2}\right)^{\prime}$ is incompatible with every member of $\widehat{f}(\mathcal{I}):=\{\widehat{f}(r): r \in \mathcal{I}\}$, so $\widehat{f}(\mathcal{I})$ is not predense in $\left(\mathbb{P}_{\lg \left(\overline{\mathbb{Q}}^{2}\right)}^{2}\right)^{\prime}$, as required.

Second, assume $\mathcal{I}$ is predense in $\left(\mathbb{P}_{\lg \left(\overline{\mathbb{Q}}^{1}\right)}^{1}\right)^{\prime}$. So for each $n, \mathcal{I}_{n}=\left\{r\left\lceil\alpha^{n}\right.\right.$ : $r \in \mathcal{I}\}$ is predense in $\left(\mathbb{P}_{\alpha_{n}}^{1}\right)^{\prime}$. Hence, by the induction hypothesis for each $n$, $\widehat{f}\left(\mathcal{I}_{n}\right)=\left\{\widehat{f}(r): r \in \mathcal{I}_{n}\right\}$ is a predense subset of $\left(\mathbb{P}_{\lg \left(\overline{\mathbb{Q}}^{2}\right)}^{2}\right)^{\prime}$.

Let $A_{n}^{1}$ be the strong $\overline{\mathbb{Q}}$-closure of $\bigcup_{p \in \mathcal{I}_{n}} \operatorname{supp}(p), A_{\omega}^{1}=\bigcup_{n<\omega} A_{n}^{1}$, and let $A_{n}^{2}$ be the strong $\overline{\mathbb{Q}}^{2}$-closure of $\bigcup_{p \in \widehat{f}\left(\mathcal{I}_{n}\right)} \operatorname{supp}(p), A_{\omega}^{2}=\bigcup_{n<\omega} A_{n}^{2}$. Thus if $k<m \leq \omega$, then $A_{k}^{1} \subseteq A_{m}^{1}$ and $A_{k}^{2} \subseteq A_{m}^{2}$, hence $\left(\mathbb{P}_{A_{k}^{1}}^{1}\right)^{\prime} \lessdot\left(\mathbb{P}_{A_{m}^{1}}^{1}\right)^{\prime}$ and $\left(\mathbb{P}_{A_{k}^{2}}^{2} \lessdot \mathbb{P}_{A_{m}^{2}}^{2}\right)^{\prime}$.

Remembering clause (g) of Definition 3.10(2), clearly

$\oplus \quad$ if $\alpha \in \bigcup_{p \in \mathcal{I}} \operatorname{supp}(p) \subseteq \operatorname{dom}\left(f_{1}\right)$, then $\bigwedge_{n}(\forall n)\left[\alpha \in A_{n}^{1} \leftrightarrow f_{1}(\alpha) \in A_{n}^{2}\right]$,

and hence for $p \in \mathcal{I}, \widehat{f}\left(p\left\lceil\alpha^{n}\right)=\widehat{f}\left(p\left\lceil A_{n}^{1}\right)=\widehat{f}(p)\left\lceil A_{n}^{2}\right.\right.\right.$. Since $p \in \mathcal{I} \rightarrow$ $\bigvee_{n}\left(p \in \mathcal{I}_{n}\right)\left(\right.$ as $\operatorname{dom}(p)$ is bounded in $\left.\lg \left(\overline{\mathbb{Q}}^{1}\right)\right)$, clearly $\widehat{f}(\mathcal{I})=\bigcup_{n<\omega} \widehat{f}\left(\mathcal{I}_{n}\right)$. Hence $q \in \mathbb{P}_{A_{\omega}^{2}}^{\prime}$ is incompatible in $\left(\mathbb{P}_{\lg \left(\overline{\mathbb{Q}}^{2}\right)}^{2}\right)^{\prime}$ with every $p \in \widehat{f}(\mathcal{I})$ iff $q$ is incompatible with every $p \in \widehat{f}\left(\mathcal{I}_{n(q)}\right)$ in $\left(\mathbb{P}_{\lg \left(\overline{\mathbb{Q}}^{2}\right)}^{2}\right)^{\prime}$, where $n(q)=\min \left\{n: q \in \mathbb{P}_{A_{n}^{2}}^{2}\right\}$, which is well defined as $\left\langle A_{m}^{2}: m \leq \omega\right\rangle$ is increasing continuous. As each $\widehat{f}\left(\mathcal{I}_{n(q)}\right)$ is predense in $\left(\mathbb{P}_{\lg \left(\overline{\mathbb{Q}}^{2}\right)}^{1}\right)^{\prime}$ (see above using the induction hypothesis), there is no such $q$. So $\widehat{f}(\mathcal{I})$ is predense in $\mathbb{P}_{A_{\omega}^{2}}^{2}$, but $A_{\omega}^{2}$ is strongly $\overline{\mathbb{Q}}^{2}$-closed (being the union of such sets), hence (by 3.8) $\left(\mathbb{P}_{A_{\omega}^{2}}^{2}\right)^{\prime} \lessdot\left(\mathbb{P}_{\lg \left(\overline{\mathbb{Q}}^{2}\right)}^{2}\right)^{\prime}$, so $\widehat{f}(\mathcal{I})$ is predense, and we have finished. 
CASE 4: $\lg \left(\overline{\mathbb{Q}}^{1}\right)=\gamma^{*}+1$. By Definition 3.1 all is translated to the $\gamma^{*}$ case (including instances of $3.11(5)$ ), so we can apply the induction hypothesis. $\mathbf{~}_{3.12}$

3.13. Claim. If $\overline{\mathcal{F}}$ witnesses $A$ for $\overline{\mathbb{Q}}$ inside $A^{\prime}$, then:

(a) $\mathbb{P}_{A}^{\prime} \lessdot \mathbb{P}_{A^{\prime}}^{\prime} \lessdot \mathbb{P}_{\lg (\overline{\mathbb{Q}})}$.

(b) $p, q \in \mathbb{P}_{A}^{\prime}$ are compatible in $\mathbb{P}_{\lg (\overline{\mathbb{Q}})}^{\prime}$ iff they are compatible in $\mathbb{P}_{A}^{\prime}$.

(c) $\mathcal{I} \subseteq \mathbb{P}_{A}^{\prime}$ is predense in $\mathbb{P}_{\lg (\overline{\mathbb{Q}})}^{\prime}$ iff it is predense in $\mathbb{P}_{A}^{\prime}$ (without loss of generality $\mathcal{I}$ is countable).

(d) There are unique $f, \overline{\mathbb{Q}}^{\prime}$ such that $\overline{\mathbb{Q}}^{\prime} \in \mathcal{K}_{\kappa}^{s}, f \in \operatorname{PAUT}\left(\overline{\mathbb{Q}}^{\prime}, \overline{\mathbb{Q}}\right), f$ is order preserving, $\operatorname{dom}(f)=\lg \left(\overline{\mathbb{Q}}^{\prime}\right)(=\operatorname{otp}(A))$ and $\operatorname{rang}(f)=A$; the essential part is: if $p, q \in \mathbb{P}_{A}^{\prime}$ and $\gamma \in \operatorname{dom}(p) \cap \operatorname{dom}(q)$, then

$$
q\left\lceil\gamma \Vdash_{\mathbb{P}_{\gamma}^{\prime}} p(\gamma) \leq_{\underset{\alpha}{\mathbb{Q}_{\gamma}}} q(\gamma) \Rightarrow q\left\lceil\gamma \Vdash_{\mathbb{P}_{\gamma \cap A}^{\prime}} p(\gamma) \leq_{\underset{\sim}{\mathbb{Q}_{\gamma}}} q(\gamma) .\right.\right.
$$

CONCLUDing REMARK. We describe below an application of the method.

3.14. Claim. Assume for simplicity $\mathbf{V}=\mathrm{GCH}$. For some c.c.c. forcing notion $\mathbb{P}$ of cardinality $\aleph_{3}$ we have, in $\mathbf{V}^{\mathbb{P}}$ :

(a) $2^{\aleph_{0}}=\aleph_{3}$.

(b) $\operatorname{add}($ meagre $)=\aleph_{1}$.

(c) $\operatorname{cov}($ meagre $)=\aleph_{2}$, moreover unif(meagre $)=\aleph_{2}$.

(d) $\mathfrak{d}=\aleph_{3}$.

3.15. REMARK. We can use other three cardinals, and use very little cardinal arithmetic assumption. with:

Proof. Let $\overline{\mathbb{Q}}=\left\langle\mathbb{P}_{i}, \mathbb{Q}_{j}, a_{j}, \eta_{i}: i \leq \omega_{3}+\omega_{2}, j<\omega_{3}+\omega_{2}\right\rangle$ be a FS iteration

- $\eta_{i}$ the generic real of $\mathbb{Q}_{j}$,

- if $j<\omega_{3}$ then $\mathbb{Q}_{j}$ is Cohen, say $(\omega>\omega, \triangleleft)$, so $\eta_{j}$ is undominated,

- if $j=\omega_{3}+\zeta$ with $\zeta<\omega_{2}$ then $\mathbb{Q}_{j}$ is Random $\mathbf{V}\left[\left\langle\eta_{i}: i \in a_{j}\right\rangle\right]$,

- if $b \subseteq a \in\left[\omega_{3}+\omega_{2}\right]^{\leq \aleph_{1}}$ then for arbitrarily large $i \in\left(\omega_{3}, \omega_{3}+\omega_{2}\right)$ we have $a \cap a_{i}=b$.

Let $\mathbb{P}=\mathbb{P}_{\omega_{3}+\omega_{2}}$. Trivially $|\mathbb{P}|=\aleph_{3}$. So in $\mathbf{V}$ we know:

(a) As $|\mathbb{P}| \leq \aleph_{3}$ the $\leq$ inequality holds; the other inequality holds as $\left\langle\eta_{i}: i<\omega_{3}\right\rangle$ is a sequence of $\aleph_{3}$ distinct reals.

(b) As in [Sh 592] we know that $\mathfrak{b}$ is not increased by $\mathbb{P}$, so $\mathfrak{b}=\aleph_{1}$ but $\operatorname{add}($ meagre $) \leq \mathfrak{b}$.

(c) If for $i<\omega_{1}$ we have Borel sets $A_{i} \subseteq \omega_{2}$ in $\mathbf{V}^{\mathbb{P}}$, then for some $\alpha<\omega_{2}$, $\left\langle A_{i}: i\left\langle\omega_{1}\right\rangle \in \mathbf{V}^{\mathbb{P}_{\omega_{3}+i}}\right.$, and the forcing $\mathbb{P}_{\omega_{3}+i+\omega} / \mathbb{P}_{\omega_{3}+i}$ adds a Cohen real over $\mathbf{V}^{\mathbb{P}_{\omega_{3}+i}}$ (as we are using FS iteration). So $\operatorname{cov}\left(\right.$ meagre) $>\aleph_{1}$ and in fact unif(meagre) $\geq \aleph_{2}$. The reverse inequality holds by the fourth $\bullet$ above. 
On the other hand, $\left\langle\eta_{\omega_{3}+i}: i<\omega_{2}\right\rangle$ is a non-null set of reals, hence unif(null $) \leq \aleph_{2}$; but cov(meagre $) \leq$ unif(null), so cov(meagre $) \leq \aleph_{2}$. So together cov(meagre $)=\aleph_{2}$.

(d) Now $\mathfrak{d} \leq 2^{\aleph_{0}}=\aleph_{3}$, on the other hand, as in [Sh 592] for no $A \in\left[\omega_{3}\right]^{\aleph_{2}}$ from $\mathbf{V}$, is there a $<^{*}$-bound to $\left\{\underset{\sim}{\eta_{i}}: i \in A\right\}$.

\section{References}

[Fe94] D. Fremlin, Problem list, circulated notes; available from http://www.essex. ac.uk/maths/staff/fremlin/measur.htm.

[GiSh 582] M. Gitik and S. Shelah, More on real-valued measurable cardinals and forcing with ideals, Israel J. Math. 124 (2001), 221-242; math.LO/9507208.

[J] T. Jech, Set Theory, Academic Press, New York, 1978.

[Ko] P. Komjáth, On second-category sets, Proc. Amer. Math. Soc. 107 (1989), 653-654.

[Sh:b] S. Shelah, Proper Forcing, Lecture Notes in Math. 940, Springer, Berlin, 1982.

[Sh:f] - Proper and Improper Forcing, Perspectives in Math. Logic, Springer, 1998.

[Sh 592] -, Covering of the null ideal may have countable cofinality, Fund. Math. 166 (2000), 109-136; math.LO/9810181.

[Sh 666] -, On what I do not understand (and have something to say), ibid., 1-82; math.LO/9906113.

Einstein Institute of Mathematics

Edmond J. Safra Campus

The Hebrew University of Jerusalem

Givat Ram, Jerusalem 91904, Israel

E-mail: shelah@math.huji.ac.il
Department of Mathematics Hill Center-Busch Campus Rutgers, The State University of New Jersey

110 Frelinghuysen Road Piscataway, NJ 08854-8019, U.S.A.

Received 28 February 2001;

in revised form 6 July 2003 